\title{
General Toeplitz operators on weighted Bloch-type spaces in the unit ball of $\mathbb{C}^{n}$
}

\section{A El-Sayed Ahmed*}

\section{"Correspondence:}

ahsayed80@hotmail.com

Mathematics Department, Faculty

of Science, Sohag University, Sohag,

82524, Egypt

Current address: Mathematics

Department, Faculty of Science, Taif University, El-Hawiah, Box 888, Taif,

Saudi Arabia

\begin{abstract}
In this paper, we consider the weighted Bloch-type spaces $\mathcal{B}_{\omega}^{\alpha, \beta}\left(\mathbb{B}_{n}\right)$ with $\alpha>0$ and $\beta \geq 0$ in the unit ball of $\mathbb{C}^{n}$. We present some basic properties of the spaces $\mathcal{B}_{\omega}^{\alpha, \beta}\left(\mathbb{B}_{n}\right)$, then we consider the Toeplitz operator $T_{\mu}^{\alpha, \beta ; \omega}$ acting between $\mathcal{B}_{\omega}^{\alpha, \beta}\left(\mathbb{B}_{n}\right)$ spaces, where $\mu$ is a positive Borel measure in the unit ball $\mathbb{B}_{n}$. Moreover, we characterize complex measures $\mu$ for which the Toeplitz operator $T_{\mu}^{\alpha, \beta ; \omega}$ is bounded or compact on $\mathcal{B}_{\omega}^{\alpha, \beta}\left(\mathbb{B}_{n}\right)$.

MSC: $47 \mathrm{~B} 35 ; 32 \mathrm{~A} 18$
\end{abstract}

Keywords: Toeplitz operators; weighted Bloch-type spaces; weighted Bergman spaces

\section{Introduction}

We start here with some terminology, notations and definitions of various classes of analytic functions defined on the unit ball of $\mathbb{C}^{n}$.

Let $\mathbb{B}_{n}$ be the unit ball of the $n$-dimensional complex Euclidean space $\mathbb{C}^{n}$. The boundary of $\mathbb{B}_{n}$ is denoted by $\mathbb{S}_{n}$ and is called the unit sphere in $\mathbb{C}^{n}$. Occasionally, we will also need the closed unit ball $\overline{\mathbb{B}}_{n}$. We denote the class of all holomorphic functions on the unit ball $\mathbb{B}_{n}$ by $\mathcal{H}\left(\mathbb{B}_{n}\right)$. The ball centered at $\mathbf{z} \in \mathbb{C}^{n}$ with radius $r$ is denoted by $B(\mathbf{z}, r)$. For $\alpha>-1$, let $d v_{\alpha}(\mathbf{z})=c_{\alpha}\left(1-|\mathbf{z}|^{2}\right)^{\alpha} d v$, where $d v$ is the normalized Lebesgue volume measure on $\mathbb{B}_{n}$ and $c_{\alpha}=\frac{\Gamma(n+\alpha+1)}{n ! \Gamma(\alpha+1)}$ (where $\Gamma$ denotes the gamma function) so that $v_{\alpha}\left(\mathbb{B}_{n}\right) \equiv 1$. The surface measure on $\mathbb{S}_{n}$ is denoted by $d \sigma$. Once again, we normalize $\sigma$ so that $\sigma_{\alpha}\left(\mathbb{S}_{n}\right) \equiv 1$. For any $\mathbf{z}=\left(z_{1}, z_{2}, \ldots, z_{n}\right), \mathbf{w}=\left(w_{1}, w_{2}, \ldots, w_{n}\right) \in \mathbb{C}^{n}$, the inner product is defined by

$$
\langle\mathbf{z}, \mathbf{w}\rangle=\sum_{k=1}^{n} z_{k} \bar{w}_{k} .
$$

For every point $\mathbf{a} \in \mathbb{B}_{n}$, the Möbius transformation $\varphi_{\mathbf{a}}: \mathbb{B}_{n} \rightarrow \mathbb{B}_{n}$ is defined by

$$
\varphi_{\mathbf{a}}(\mathbf{z})=\frac{\mathbf{a}-P_{\mathbf{a}}(\mathbf{z})-S_{\mathbf{a}} Q_{\mathbf{a}}(\mathbf{z})}{1-\langle\mathbf{z}, \mathbf{a}\rangle}, \quad \mathbf{z} \in \mathbb{B}_{n},
$$

where $S_{\mathbf{a}}=\sqrt{1-|\mathbf{a}|^{2}}, P_{\mathbf{a}}(\mathbf{z})=\frac{\mathbf{a}(\mathbf{z}, \mathbf{a}\rangle}{|\mathbf{a}|^{2}}, P_{0}=0$ and $Q_{\mathbf{a}}=I-P_{\mathbf{a}}$. The map $\varphi_{\mathbf{a}}$ has the following properties that $\varphi_{\mathbf{a}}(0)=\mathbf{a}, \varphi_{\mathbf{a}}(\mathbf{a})=0, \varphi_{\mathbf{a}}=\varphi_{\mathbf{a}}^{-1}$ and

$$
1-\left\langle\varphi_{\mathbf{a}}(\mathbf{z}), \varphi_{\mathbf{a}}(\mathbf{w})\right\rangle=\frac{\left(1-|\mathbf{a}|^{2}\right)(1-\langle\mathbf{z}, \mathbf{w}\rangle)}{(1-\langle\mathbf{z}, \mathbf{a}\rangle)(1-\langle\mathbf{a}, \mathbf{w}\rangle)}
$$

(0) 2013 El-Sayed Ahmed; licensee Springer. This is an Open Access article distributed under the terms of the Creative Commons Attribution License (http://creativecommons.org/licenses/by/2.0), which permits unrestricted use, distribution, and reproduction in any medium, provided the original work is properly cited. 
where $\mathbf{z}$ and $\mathbf{w}$ are arbitrary points in $\mathbb{B}_{n}$. In particular,

$$
1-\left|\varphi_{\mathbf{a}}(\mathbf{z})\right|^{2}=\frac{\left(1-|\mathbf{a}|^{2}\right)\left(1-|\mathbf{z}|^{2}\right)}{|1-\langle\mathbf{z}, \mathbf{a}\rangle|^{2}} .
$$

For $f \in \mathcal{H}\left(\mathbb{B}_{n}\right)$, the holomorphic gradient of $f$ at $\mathbf{z}$ is defined by

$$
\nabla f(\mathbf{z})=\left(\frac{\partial f}{\partial z_{1}}(\mathbf{z}), \frac{\partial f}{\partial z_{2}}(\mathbf{z}), \ldots, \frac{\partial f}{\partial z_{n}}(\mathbf{z})\right)
$$

and the radial derivative of $f$ at $\mathbf{z}$ is defined by

$$
\mathfrak{R} f(\mathbf{z})=\langle\nabla f, \overline{\mathbf{z}}\rangle=\sum_{j=1}^{n} z_{j} \frac{\partial f(\mathbf{z})}{\partial z_{j}} .
$$

Similarly, the Möbius invariant complex gradient of $f$ at $\mathbf{z}$ is defined by

$$
\widetilde{\nabla} f(\mathbf{z})=\nabla\left(f \circ \varphi_{\mathbf{z}}\right)(0) .
$$

For $\alpha>0$, a function $f \in \mathcal{H}\left(\mathbb{B}_{n}\right)$ is said to belong to the $\alpha$-Bloch spaces $\mathcal{B}^{\alpha}\left(\mathbb{B}_{n}\right)$ if (see [1])

$$
b_{\alpha}(f)\left(\mathbb{B}_{n}\right)=\sup _{\mathbf{z} \in \mathbb{B}_{n}}|\nabla f(\mathbf{z})|\left(1-|\mathbf{z}|^{2}\right)^{\alpha}<\infty .
$$

The little Bloch space $\mathcal{B}_{0}^{\alpha}\left(\mathbb{B}_{n}\right)$ consists of all $f \in \mathcal{B}^{\alpha}\left(\mathbb{B}_{n}\right)$ such that

$$
\lim _{|\mathbf{z}| \rightarrow 1^{-}}|\nabla f(\mathbf{z})|\left(1-|\mathbf{z}|^{2}\right)^{\alpha}=0 .
$$

With the norm $\|f\|_{\mathcal{B}^{\alpha}}=|f(0)|+b_{\alpha}(f)\left(\mathbb{B}_{n}\right)$, we know that $\mathcal{B}^{\alpha}\left(\mathbb{B}_{n}\right)$ becomes a Banach space and $\mathcal{B}_{0}^{\alpha}\left(\mathbb{B}_{n}\right)$ is its closed subspace (see [1]). For $\alpha=1$, the spaces $\mathcal{B}^{1}\left(\mathbb{B}_{n}\right)$ and $\mathcal{B}_{0}^{1}\left(\mathbb{B}_{n}\right)$ become the Bloch and the little Bloch space, respectively (see, for example, [2-5]). Zhu in [5] says that the norm $\|f\|_{\mathcal{B}\left(\mathbb{B}_{n}\right)}$ is equivalent to

$$
|f(0)|+\sup _{\mathbf{z} \in \mathbb{B}_{n}}|\Re f(\mathbf{z})|\left(1-|\mathbf{z}|^{2}\right) .
$$

For $\alpha>-1$ and $0<p<\infty$, the weighted Bergman space $A_{\alpha}^{p}\left(\mathbb{B}_{n}\right)$ consists of holomorphic functions $f \in L^{p}\left(\mathbb{B}_{n}, d v_{\alpha}\right)$ such that

$$
\|f\|_{A_{\alpha}^{p}\left(\mathbb{B}_{n}\right)}^{p}:=\int_{\mathbb{B}_{n}}|f(\mathbf{z})|^{p} d v_{\alpha}(\mathbf{z})<\infty,
$$

that is, $A_{\alpha}^{p}\left(\mathbb{B}_{n}\right)=L^{p}\left(\mathbb{B}_{n}, d v_{\alpha}\right) \cap \mathcal{H}\left(\mathbb{B}_{n}\right)$. When the weight $\alpha=0$, we simply write $A^{p}\left(\mathbb{B}_{n}\right)$ for $A_{0}^{p}\left(\mathbb{B}_{n}\right)$. In the special case when $p=2, A_{\alpha}^{2}\left(\mathbb{B}_{n}\right)$ is a Hilbert space. It is well known that for $\alpha>-1$, the Bergman kernel of $A_{\alpha}^{2}\left(\mathbb{B}_{n}\right)$ is given by

$$
K^{\alpha}(\mathbf{z}, \mathbf{w})=\frac{1}{(1-\langle\mathbf{z}, \mathbf{w}\rangle)^{n+\alpha+1}}, \quad \mathbf{z}, \mathbf{w} \in \mathbb{B}_{n} .
$$


For $\alpha>-1$, a complex measure $\mu$ is such that

$$
\left|\int_{\mathbb{B}_{n}}\left(1-|\mathbf{w}|^{2}\right)^{\alpha} d \mu(\mathbf{w})\right|=\left|\int_{\mathbb{B}_{n}} d \mu_{\alpha}(\mathbf{w})\right|<\infty .
$$

The general Bergman projection $P_{\alpha}$ is the orthogonal projection of the measure $\mu$ from $L^{2}\left(\mathbb{B}_{n}, d v_{\alpha}\right)$ into $A_{\alpha}^{2}\left(\mathbb{B}_{n}\right)$ defined by

$$
P_{\alpha}(\mu)(\mathbf{z})=c_{\alpha} \int_{\mathbb{B}_{n}} \frac{\left(1-|\mathbf{w}|^{2}\right)^{\alpha}}{(1-\langle\mathbf{z}, \mathbf{w}\rangle)^{n+\alpha+1}} d \mu(\mathbf{w})=c_{\alpha} \int_{\mathbb{B}_{n}} \frac{d \mu_{\alpha}(\mathbf{w})}{(1-\langle\mathbf{z}, \mathbf{w}\rangle)^{n+\alpha+1}} .
$$

The general Bergman projection of the function $f$ is

$$
P_{\alpha} f(\mathbf{z})=c_{\alpha} \int_{\mathbb{B}_{n}} \frac{f(\mathbf{w})\left(1-|\mathbf{w}|^{2}\right)^{\alpha}}{(1-\langle\mathbf{z}, \mathbf{w}\rangle)^{n+\alpha+1}} d v(\mathbf{w})=c_{\alpha} \int_{\mathbb{B}_{n}} \frac{f(\mathbf{w}) d v_{\alpha}(\mathbf{w})}{(1-\langle\mathbf{z}, \mathbf{w}\rangle)^{n+\alpha+1}} .
$$

Let $\omega:(0,1] \rightarrow(0, \infty)$ be a right-continuous and nondecreasing function. For a complex measure $\mu, \alpha>-1, \beta \geq 0$, and $f \in L^{1}\left(\mathbb{B}_{n}, d \nu_{\alpha+\beta}\right)$, define weighted general Toeplitz operator as follows:

$$
\begin{aligned}
T_{\mu}^{\alpha, \beta ; \omega} f(\mathbf{z}) & =\frac{c_{\alpha+\beta}}{\left(1-\left|\varphi_{\mathbf{z}}(\mathbf{w})\right|^{2}\right)^{\beta} \omega(1-|\mathbf{w}|)} \int_{\mathbb{B}_{n}} \frac{\left(1-|\mathbf{w}|^{2}\right)^{\alpha+\beta} f(\mathbf{w})}{(1-\langle\mathbf{z}, \mathbf{w}\rangle)^{n+\alpha+\beta+1}} d \mu(\mathbf{w}) \\
& =\frac{c_{\alpha+\beta}}{\left(1-\left|\varphi_{\mathbf{z}}(\mathbf{w})\right|^{2}\right)^{\beta} \omega(1-|\mathbf{w}|)} \int_{\mathbb{B}_{n}} \frac{f(\mathbf{w}) d \mu_{\alpha+\beta}(\mathbf{w})}{(1-\langle\mathbf{z}, \mathbf{w}\rangle)^{n+\alpha+\beta+1}} .
\end{aligned}
$$

Thus $P_{\alpha+\beta ; \omega}(\mu)(\mathbf{z})=T_{\mu}^{\alpha, \beta ; \omega}(1)(\mathbf{z})$, where 1 stands for a constant function.

Toeplitz operators have been studied extensively on the Bergman spaces by many authors. For references, see [6] and [7]. Boundedness and compactness of the general Toeplitz operators $T_{\mu}^{\alpha}$ on the $\alpha$-Bloch $\mathcal{B}^{\alpha}(\mathbb{D})$ spaces have been investigated in [8] on the unit disk $\mathbb{D}$ for $0<\alpha<\infty$. Also, in [9], the authors extended the general Toeplitz operator $T_{\mu}^{\alpha}$ to $\mathcal{B}^{\alpha}\left(\mathbb{B}_{n}\right)$ with $1 \leq \alpha<2$. Recently, in [10], the general Toeplitz operators $T_{\mu}^{\alpha}$ on the analytic Besov $B_{p}(\mathbb{D})$ spaces with $1 \leq p<\infty$ have been investigated. Under a prerequisite condition, the authors characterized a complex measure $\mu$ on the unit disk for which $T_{\mu}^{\alpha}$ is bounded or compact on the Besov space $B_{p}(\mathbb{D})$. For more studies on the Toeplitz operator, we refer to [11-17].

In this paper, we consider the weighted Bloch-type spaces $\mathcal{B}_{\omega}^{\alpha, \beta}\left(\mathbb{B}_{n}\right)$ with $\alpha>0$ and $\beta \geq 0$ in the unit ball of $\mathbb{C}^{n}$. We prove a certain integral representation theorem that is used to determine the degree of growth of the functions in the space $\mathcal{B}_{\omega}^{\alpha, \beta}\left(\mathbb{B}_{n}\right)$. It is also proved that the space $\mathcal{B}_{\omega}^{\alpha, \beta}\left(\mathbb{B}_{n}\right)$ is a Banach space for each weight $\alpha>0, \beta \geq 0$, and the Banach dual of the Bergman space $A^{1}\left(\mathbb{B}_{n}\right)$ is $\mathcal{B}_{\omega}^{\alpha, \beta}\left(\mathbb{B}_{n}\right)$ for each $\alpha \geq 1, \beta \geq 0$. Further, we extend the Toeplitz operator $T_{\mu}^{\alpha, \beta ; \omega}$ to $\mathcal{B}_{\omega}^{\alpha, \beta}\left(\mathbb{B}_{n}\right)$ in the unit ball of $\mathbb{C}^{n}$ and completely characterize the positive Borel measure $\mu$ such that $T_{\mu}^{\alpha, \beta ; \omega}$ is bounded or compact in $\mathcal{B}_{\omega}^{\alpha, \beta}\left(\mathbb{B}_{n}\right)$ spaces with $\alpha+\beta \geq 1$.

Throughout the paper, we say that the expressions $A$ and $B$ are equivalent, and write $A \approx$ $B$ whenever there exist positive constants $C_{1}$ and $C_{2}$ such that $C_{1} A \leq B \leq C_{2} A$. As usual, the letter $C$ denotes a positive constant, possibly different on each occurrence. Hereafter, $\omega$ stands for a right-continuous and nondecreasing function. 
Theorem 1.1 (see [5, Theorem 1.12]) Suppose $b$ is real and $s>-1$. Then the integrals

$$
I_{b}(\mathbf{z})=\int_{\mathbb{S}_{n}} \frac{d \sigma(\zeta)}{|1-\langle\mathbf{z}, \zeta\rangle|^{n+b}}, \quad \mathbf{z} \in \mathbb{B}_{n}
$$

and

$$
J_{b, s}(\mathbf{z})=\int_{\mathbb{B}_{n}} \frac{\left(1-|\mathbf{w}|^{2}\right)^{s} d v(\mathbf{w})}{|1-\langle\mathbf{z}, \mathbf{w}\rangle|^{n+1+s+b}}, \quad \mathbf{z} \in \mathbb{B}_{n},
$$

have the following asymptotic properties.

(1) If $b<0$, then $I_{b}(\mathbf{z})$ and $J_{b, s}(\mathbf{z})$ are both bounded in $\mathbb{B}_{n}$.

(2) If $b=0$, then

$$
I_{b}(\mathbf{z}) \approx I_{b, s}(\mathbf{z}) \approx \log \frac{1}{1-|\mathbf{z}|^{2}} \quad \text { as }|\mathbf{z}| \rightarrow 1^{-1} .
$$

(3) If $b>0$, then

$$
I_{b}(\mathbf{z}) \approx J_{b, s}(\mathbf{z}) \approx\left(1-|\mathbf{z}|^{2}\right)^{-b} \text { as }|\mathbf{z}| \rightarrow 1^{-1} .
$$

Lemma 1.1 (see [5, Lemma 3.3]) Suppose $\gamma$ is a real constant and $g \in L^{1}\left(\mathbb{B}_{n}, d v\right)$. If

$$
u(\mathbf{z})=\left(1-\left|\varphi_{\mathbf{a}}(\mathbf{z})\right|^{2}\right)^{\beta} \int_{\mathbb{B}_{n}} \frac{g(\mathbf{w}) d v(\mathbf{w})}{(1-\langle\mathbf{z}, \mathbf{w}\rangle)^{\gamma}}, \quad \mathbf{z} \in \mathbb{B}_{n},
$$

then

$$
|\widetilde{\nabla} u(\mathbf{z})| \leq \sqrt{2}|\gamma|\left(1-|\mathbf{z}|^{2}\right)^{\frac{1}{2}} \int_{\mathbb{B}_{n}} \frac{g(\mathbf{w}) d \nu(\mathbf{w})}{|1-\langle\mathbf{z}, \mathbf{w}\rangle|^{\gamma+\frac{1}{2}}}, \quad \forall \mathbf{z} \in \mathbb{B}_{n} .
$$

Let $\beta(\cdot, \cdot)$ be the Bergman metric on $\mathbb{B}_{n}$. Denote the Bergman metric ball at $\mathbf{w}^{(j)}$ by $B\left(\mathbf{w}^{(j)}, r\right)=\left\{\mathbf{z} \in \mathbb{B}_{n}: \beta\left(\mathbf{w}^{(j)}, \mathbf{z}\right)<r\right\}$, where $\mathbf{w}^{(j)} \in \mathbb{B}_{n}$ and $r>0$.

Lemma 1.2 (see [5, Theorem 2.23]) For fixed $r>0$, there is a sequence $\left\{\mathbf{w}^{(j)}\right\} \in \mathbb{B}_{n}$ such that:

- $\bigcup_{j=1}^{\infty} B\left(\mathbf{w}^{(j)}, r\right)=\mathbb{B}_{n}$;

- there is a positive integer $N$ such that each $\mathbf{z} \in \mathbb{B}_{n}$ is contained in at most $N$ of the sets $B\left(\mathbf{w}^{(j)}, 2 r\right)$.

The following characterization of Carleson measures can be found in [6], or in [5].

A positive Borel measure $\mu$ on the unit ball $\mathbb{B}_{n}$ is said to be a Carleson measure for the Bergman space $A_{\alpha}^{p}\left(\mathbb{B}_{n}\right)$ if

$$
\int_{\mathbb{B}_{n}}|f(\mathbf{z})|^{p} d v_{\alpha}(\mathbf{z}) \leq C\|f\|_{A_{\alpha}^{p}\left(\mathbb{B}_{n}\right)}^{p}, \quad \forall f \in A_{\alpha}^{p}\left(\mathbb{B}_{n}\right) .
$$

It is well known that a positive Borel measure $\mu$ is a Carleson measure if and only if there is a positive constant $C$ such that

$$
\sup _{\mathbf{w}^{(j)} \in \mathbb{B}_{n}} \frac{\mu\left(B\left(\mathbf{w}^{(j)}, r\right)\right)}{v\left(B\left(\mathbf{w}^{(j)}, r\right)\right)}<\infty
$$


where $\left\{\mathbf{w}^{(j)}\right\}$ is the sequence in Lemma 1.2. If $\mu$ satisfies that

$$
\lim _{j \rightarrow \infty} \frac{\mu\left(B\left(\mathbf{w}^{(j)}, r\right)\right)}{v\left(B\left(\mathbf{w}^{(j)}, r\right)\right)}=0
$$

then $\mu$ is called a vanishing Carleson measure.

For a given reasonable function $\omega:(0,1] \rightarrow(0, \infty)$, the weighted Bloch space $\mathcal{B}_{\omega}$ of several complex variables is defined as the set of all analytic functions $f$ on $\mathbb{B}_{n}$ satisfying

$$
(1-|\mathbf{z}|)^{\alpha}|\nabla f(\mathbf{z})| \leq C \omega(1-|\mathbf{z}|), \quad \mathbf{z} \in \mathbb{B}_{n} \text {, where } \alpha \in(0, \infty)
$$

for some fixed $C=C_{f}>0$. In the special case where $\omega \equiv 1, \mathcal{B}_{\omega}$ reduces to the classical Bloch space $\mathcal{B}$ in $\mathbb{C}^{n}$. This class of functions extends and generalizes the well known Bloch space. Now, we define the space $\mathcal{B}_{\alpha, \beta ; \omega}\left(\mathbb{B}_{n}\right)$ in the unit ball $\mathbb{B}_{n}$. For $\alpha>0$ and $\beta \geq 0$, a function $f \in \mathcal{H}\left(\mathbb{B}_{n}\right)$ is said to belong to the $(\alpha, \beta ; \omega)$-Bloch space $\mathcal{B}_{\alpha, \beta ; \omega}\left(\mathbb{B}_{n}\right)$ if

$$
b_{\alpha, \beta ; \omega}(f)\left(\mathbb{B}_{n}\right)=\sup _{\mathbf{a}, \mathbf{z} \in \mathbb{B}_{n}} \frac{\left(1-|\mathbf{z}|^{2}\right)^{\alpha+\beta}}{\left(1-\left|\varphi_{\mathbf{a}}(\mathbf{z})\right|^{2}\right)^{\beta} \omega(1-|\mathbf{z}|)}|\nabla f(\mathbf{z})|<\infty .
$$

The little $(\alpha, \beta ; \omega)$-Bloch space $\mathcal{B}_{\alpha, \beta ; \omega, 0}\left(\mathbb{B}_{n}\right)$ is a subspace of $\mathcal{B}_{\alpha, \beta ; \omega}\left(\mathbb{B}_{n}\right)$ consisting of all $f \in$ $\mathcal{B}_{\alpha, \beta ; \omega}\left(\mathbb{B}_{n}\right)$ such that

$$
\lim _{|\mathbf{a}| \rightarrow 1^{-}} \lim _{|\mathbf{z}| \rightarrow 1^{-}} \frac{\left(1-|\mathbf{z}|^{2}\right)^{\alpha+\beta}}{\left(1-\left|\varphi_{\mathbf{a}}(\mathbf{z})\right|^{2}\right)^{\beta} \omega(1-|\mathbf{z}|)}|\nabla f(\mathbf{z})|=0 .
$$

If $\beta=0, \omega(1-|\mathbf{z}|)=1$, then we get the $\alpha$-Bloch space $\mathcal{B}^{\alpha}\left(\mathbb{B}_{n}\right)$ and the little $\alpha$-Bloch space $\mathcal{B}_{0}^{\alpha}\left(\mathbb{B}_{n}\right)$. If $\omega(1-|\mathbf{z}|)=1, \alpha=1$ and $\beta=0$, then we get the classical Bloch space $\mathcal{B}\left(\mathbb{B}_{n}\right)$ and $\mathcal{B}_{0}\left(\mathbb{B}_{n}\right)$. These classes extend the weighted Bloch spaces defined in [18] to the setting of several complex variables.

The logarithmic $(\alpha, \beta ; \omega)$-Bloch space $\mathcal{L} \mathcal{B}_{\omega}^{\alpha, \beta}\left(\mathbb{B}_{n}\right)$ is the space of holomorphic functions $f$ such that

$$
\sup _{\mathbf{a}, \mathbf{z} \in \mathbb{B}_{n}} \frac{\left(1-|\mathbf{z}|^{2}\right)^{\alpha+\beta}}{\left(1-\left|\varphi_{\mathbf{a}}(\mathbf{z})\right|^{2}\right)^{\beta} \omega(1-|\mathbf{z}|)}\left(\ln \frac{2}{1-|\mathbf{z}|^{2}}\right)|\nabla f(\mathbf{z})|<\infty .
$$

Correspondingly, the little logarithmic $(\alpha, \beta ; \omega)$-Bloch space $\mathcal{L B}_{\omega ; 0}^{\alpha, \beta}\left(\mathbb{B}_{n}\right)$ is a subspace of $\mathcal{L B}_{\omega}^{\alpha, \beta}\left(\mathbb{B}_{n}\right)$ consisting of all functions $f$ such that

$$
\lim _{|\mathbf{a}| \rightarrow 1^{-}} \lim _{|\mathbf{z}| \rightarrow 1^{-}} \frac{\left(1-|\mathbf{z}|^{2}\right)^{\alpha+\beta}}{\left(1-\left|\varphi_{\mathbf{a}}(\mathbf{z})\right|^{2}\right)^{\beta} \omega(1-|\mathbf{z}|)}\left(\ln \frac{2}{1-|\mathbf{z}|^{2}}\right)|\nabla f(\mathbf{z})|=0 .
$$

If $\omega(1-|\mathbf{z}|)=1$ and $\beta=0$, then we get the logarithmic $\alpha$-Bloch space $\mathcal{L} \mathcal{B}^{\alpha}\left(\mathbb{B}_{n}\right)$ and the little logarithmic $\alpha$-Bloch space $\mathcal{L} \mathcal{B}_{0}^{\alpha}\left(\mathbb{B}_{n}\right)$. If $\omega(1-|\mathbf{z}|)=1, \alpha=1$ and $\beta=0$, then we get the logarithmic Bloch space $\mathcal{L B}\left(\mathbb{B}_{n}\right)$ and $\mathcal{L} \mathcal{B}_{0}\left(\mathbb{B}_{n}\right)$ (see [19]).

\section{Holomorphic $(\alpha, \beta ; \omega)$-Bloch space in the unit ball}

In this section, we study the general $(\alpha, \beta ; \omega)$-Bloch space $\mathcal{B}_{\omega}^{\alpha, \beta}\left(\mathbb{B}_{n}\right)$ in the unit ball of $\mathbb{C}^{n}$ by giving some characterizations of $(\alpha, \beta ; \omega)$-Bloch space, then we present several auxiliary results, which play important roles in the proofs of our main results. 
Lemma 2.1 Let $\alpha, \beta \in(0, \infty)$ and $f \in \mathcal{B}_{\omega}^{\alpha, \beta}\left(\mathbb{B}_{n}\right)$. Suppose that

$$
\int_{0}^{1} \frac{\omega(1-t|\mathbf{z}|)|\mathbf{z}| d t}{\left(1-t^{2}|\mathbf{z}|^{2}\right)^{\alpha+\beta}}<\infty
$$

Then

$$
|f(z)| \leq|f(0)|+\|f\|_{\mathcal{B}_{\omega}^{\alpha, \beta}\left(\mathbb{B}_{n}\right)} .
$$

Proof Let $\mathbf{z} \in \mathbb{B}_{n}, 0 \leq t<1$ and $f \in \mathcal{B}_{\omega}^{\alpha, \beta}\left(\mathbb{B}_{n}\right)$. By the definition of $\mathcal{B}_{\omega}^{\alpha, \beta}\left(\mathbb{B}_{n}\right)$ and $|\mathbf{z}|>\frac{1}{2}$, we have that

$$
\begin{aligned}
\left|f(\mathbf{z})-f\left(\frac{\mathbf{z}}{2}\right)\right| & =\left|\int_{\frac{1}{2}}^{1}\langle\nabla f(t \mathbf{z}), \mathbf{z}\rangle d t\right| \\
& \leq\left|\int_{\frac{1}{2}}^{1} \Re f(t \mathbf{z}) \frac{d t}{t}\right| \\
& \leq b_{\alpha, \beta ; \omega}(f) \int_{0}^{1} \frac{\left(1-\left|\varphi_{\mathbf{a}}(t \mathbf{z})\right|^{2}\right)^{\beta} \omega(1-t|\mathbf{z}|)}{\left(1-t^{2}|\mathbf{z}|^{2}\right)^{\alpha+\beta}}|\mathbf{z}| d t \\
& \leq b_{\alpha, \beta ; \omega}(f) \int_{0}^{1} \frac{\left(1-|\mathbf{a}|^{2}\right)^{\beta} \omega(1-t|\mathbf{z}|)}{|1-\langle t \mathbf{z}, \mathbf{a}\rangle|^{2 \beta}\left(1-t^{2}|\mathbf{z}|^{2}\right)^{\alpha}}|\mathbf{z}| d t .
\end{aligned}
$$

Since $(1-|\mathbf{a}|) \leq|1-\langle t \mathbf{z}, \mathbf{a}\rangle|$ and $(1-t|\mathbf{z}|) \leq|1-\langle t \mathbf{z}, \mathbf{a}\rangle|, \mathbf{a}, \mathbf{z} \in \mathbb{B}_{n}$, we get

$$
\begin{aligned}
\left|f(\mathbf{z})-f\left(\frac{\mathbf{z}}{2}\right)\right| & \leq b_{\alpha, \beta ; \omega}(f) \int_{0}^{1} \frac{\left(1-|\mathbf{a}|^{2}\right)^{\beta} \omega(1-|\mathbf{z}|)}{(1-|\mathbf{a}|)^{\beta}(1-t|\mathbf{z}|)^{\beta}\left(1-t^{2}|\mathbf{z}|^{2}\right)^{\alpha}}|\mathbf{z}| d t \\
& \leq 4^{\beta} b_{\alpha, \beta}(f) \int_{0}^{1} \frac{\omega(1-t|\mathbf{z}|)|\mathbf{z}| d t}{\left(1-t^{2}|\mathbf{z}|^{2}\right)^{\alpha+\beta}}
\end{aligned}
$$

from which the result follows.

Theorem 2.1 For each $0<\alpha, \beta<\infty, \gamma>-1$ and $f \in \mathcal{H}\left(\mathbb{B}_{n}\right)$. Then the following conditions are equivalent:

(i) $f \in \mathcal{B}_{\omega}^{\alpha, \beta}\left(\mathbb{B}_{n}\right)$;

(ii) The function $\frac{\left(1-|\mathbf{z}|^{2}\right)^{\alpha+\beta}}{\left(1-\left|\varphi_{\mathbf{z}}(\mathbf{w})\right|^{2}\right)^{\beta} \omega(1-|\mathbf{w}|)}|\mathfrak{R} f(\mathbf{z})|$ is bounded in $\mathbb{B}_{n}$;

(iii) There exists a function $g \in L^{\infty}\left(\mathbb{B}_{n}\right)$ such that

$$
f(\mathbf{z})=\left(1-\left|\varphi_{\mathbf{z}}(\mathbf{w})\right|^{2}\right)^{\beta} \omega(1-|\mathbf{w}|) \int_{\mathbb{B}_{n}} \frac{g(\mathbf{w}) d v_{\gamma}(\mathbf{w})}{(1-\langle\mathbf{z}, \mathbf{w}\rangle)^{n+\alpha+\beta+\gamma}}, \quad \mathbf{z} \in \mathbb{B}_{n} .
$$

Proof By the Cauchy-Schwarz inequality in $\mathbb{C}^{n}$, we have

$$
|\Re f(\mathbf{z})| \leq|\mathbf{z}||\nabla f(\mathbf{z})| \leq|\nabla f(\mathbf{z})| .
$$

This proves that (i) $\Rightarrow$ (ii).

If (ii) holds, then the function

$$
g(\mathbf{z})=\frac{c_{\alpha+\beta+\gamma}}{c_{\gamma}} \frac{\left(1-|\mathbf{z}|^{2}\right)^{\alpha+\beta}}{\left(1-\left|\varphi_{\mathbf{z}}(\mathbf{w})\right|^{2}\right)^{\beta} \omega(1-|\mathbf{w}|)}\left(f(\mathbf{z})+\frac{\mathfrak{R} f(\mathbf{z})}{n+\alpha+\beta+\gamma}\right)
$$


is bounded in $\mathbb{B}_{n}$. For $\mathbf{z} \in \mathbb{B}_{n}$ consider the holomorphic function

$$
\begin{aligned}
F(\mathbf{z}) & =\int_{\mathbb{B}_{n}} \frac{g(\mathbf{w})\left(1-\left|\varphi_{\mathbf{z}}(\mathbf{w})\right|^{2}\right)^{\beta} \omega(1-|\mathbf{w}|) d \nu_{\gamma}(\mathbf{w})}{(1-\langle\mathbf{z}, \mathbf{w}\rangle)^{n+\alpha+\beta+\gamma}} \\
& =\int_{\mathbb{B}_{n}} \frac{\omega(1-|\mathbf{w}|)}{(1-\langle\mathbf{z}, \mathbf{w}\rangle)^{n+\alpha+\beta+\gamma}}\left(f(\mathbf{w})+\frac{\mathfrak{R} f(\mathbf{w})}{n+\alpha+\beta+\gamma}\right) d v_{\alpha+\beta+\gamma}(\mathbf{w}) .
\end{aligned}
$$

As in the proof of Theorem 7.1 in [5], we have $F=f$.

This shows that (ii) implies (iii). That (iii) implies (i) follows from differentiating under the integral sign and then applying Theorem 1.12 in [5].

Theorem 2.2 For each $\alpha>0, \beta \geq 0, \alpha+\beta>0$ and $s=\alpha+\beta-1$. If $s>-1$, then the Banach dual of $A^{1}\left(\mathbb{B}_{n}\right)$ can be identified with $\mathcal{B}_{\omega}^{\alpha, \beta}\left(\mathbb{B}_{n}\right)$ (with equivalent norms) under the following integral pairing:

$$
\langle f, g\rangle_{s}=\int_{\mathbb{B}_{n}} f(\mathbf{z}) \overline{g(\mathbf{z})} d v_{s}(\mathbf{z}), \quad f \in A^{1}\left(\mathbb{B}_{n}\right), g \in \mathcal{B}_{\omega}^{\alpha, \beta}\left(\mathbb{B}_{n}\right) .
$$

Proof It is easy to see that $1-(\alpha+\beta)+s>-1$. If $g \in \mathcal{B}_{\omega}^{\alpha, \beta}\left(\mathbb{B}_{n}\right)$, then by Theorem 2.1, there exists a function $h \in L^{\infty}\left(\mathbb{B}_{n}\right)$ such that

$$
g(\mathbf{z})=\frac{1}{\left(1-\left|\varphi_{\mathbf{z}}(\mathbf{w})\right|^{2}\right)^{\beta} \omega(1-|\mathbf{w}|)} \int_{\mathbb{B}_{n}} \frac{h(\mathbf{w}) d \nu_{1-(\alpha+\beta)+s}(\mathbf{w})}{(1-\langle\mathbf{z}, \mathbf{w}\rangle)^{n+1+s}}, \quad \mathbf{z}, \mathbf{w} \in \mathbb{B}_{n},
$$

and $\|h\|_{\infty} \leq C\|g\|_{\mathcal{B}_{\omega}^{\alpha, \beta}\left(\mathbb{B}_{n}\right)}$, where $C$ is a positive constant independent of $g$. By Fubini's theorem,

$$
\begin{aligned}
\langle f, g\rangle_{s} & =\int_{\mathbb{B}_{n}} f(\mathbf{z}) \overline{h(\mathbf{z})}\left(1-|\mathbf{z}|^{2}\right) d \nu_{1-(\alpha+\beta)+s}(\mathbf{z}) \\
& =c_{1-(\alpha+\beta)+s} \int_{\mathbb{B}_{n}} f(\mathbf{z}) \overline{h(\mathbf{z})} d \nu(\mathbf{z})
\end{aligned}
$$

Applying Lemma 2.15 in [5] for all $f \in A^{1}\left(\mathbb{B}_{n}\right)$, we have

$$
\int_{\mathbb{B}_{n}}|f(\mathbf{z})| d v(\mathbf{z}) \leq\|f\|_{A^{1}\left(\mathbb{B}_{n}\right)}
$$

Combining this, we see that

$$
\left|\langle f, g\rangle_{s}\right| \leq\|h\|_{\infty}\|f\|_{A^{1}\left(\mathbb{B}_{n}\right)} \leq C\|g\|_{\mathcal{B}_{\omega}^{\alpha, \beta}\left(\mathbb{B}_{n}\right)}\|f\|_{A^{1}\left(\mathbb{B}_{n}\right)} .
$$

Conversely, if $F$ is a bounded linear functional on $A^{1}\left(\mathbb{B}_{n}\right)$ and $f \in A^{1}\left(\mathbb{B}_{n}\right)$, then

$$
f_{r}(\mathbf{z})=\frac{1}{\left(1-\left|\varphi_{\mathbf{z}}(\mathbf{w})\right|^{2}\right)^{\beta} \omega(1-|\mathbf{w}|)} \int_{\mathbb{B}_{n}} \frac{f_{r}(\mathbf{w}) d v_{s}(\mathbf{w})}{(1-\langle\mathbf{z}, \mathbf{w}\rangle)^{n+1+s}} \quad \text { for } 0<r<1 .
$$

It is easy to verify (using the homogeneous expansion of the kernel function) that

$$
F\left(f_{r}\right)=\int_{\mathbb{B}_{n}} f_{r}(\mathbf{w}) F_{\mathbf{z}}\left[\frac{1}{(1-\langle\mathbf{z}, \mathbf{w}\rangle)^{n+1+s}\left(1-\left|\varphi_{\mathbf{z}}(\mathbf{w})\right|^{2}\right)^{\beta} \omega(1-|\mathbf{w}|)}\right] d v_{s}(\mathbf{w}) .
$$


Define a function $g$ on $\mathbb{B}_{n}$ by

$$
\overline{g(\mathbf{w})}=F_{\mathbf{z}}\left[\frac{1}{(1-\langle\mathbf{z}, \mathbf{w}\rangle)^{n+1+s}\left(1-\left|\varphi_{\mathbf{z}}(\mathbf{w})\right|^{2}\right)^{\beta} \omega(1-|\mathbf{w}|)}\right] .
$$

Then

$$
F\left(f_{r}\right)=\int_{\mathbb{B}_{n}} f_{r}(\mathbf{w}) \overline{g(\mathbf{w})} d v_{s}(\mathbf{w})=\langle f, g\rangle_{s} .
$$

It remains for us to show that $g \in \mathcal{B}_{\omega}^{\alpha, \beta}$.

We interchange differentiation and the application of $F$, which can be justified by using the homogeneous expansion of the kernel. The result is

$$
\overline{\Re g(\mathbf{w})}=(n+1+s) F_{\mathbf{z}}\left[\frac{1}{(1-\langle\mathbf{z}, \mathbf{w}\rangle)^{n+1+s}\left(1-\left|\varphi_{\mathbf{z}}(\mathbf{w})\right|^{2}\right)^{\beta} \omega(1-|\mathbf{w}|)}\right] .
$$

Since $F$ is bounded on $A^{1}\left(\mathbb{B}_{n}\right)$, we have

$$
|\Re g(\mathbf{w})| \leq \frac{C\|F\|}{\left(1-\left|\varphi_{\mathbf{z}}(\mathbf{w})\right|^{2}\right)^{\beta} \omega(1-|\mathbf{w}|)} \int_{\mathbb{B}_{n}} \frac{d v(\mathbf{w})}{|1-\langle\mathbf{z}, \mathbf{w}\rangle|^{n+2+s}}
$$

An application of Theorem 1.1 for $s+1=\alpha+\beta$ then shows that

$$
|\mathfrak{R} g(\mathbf{w})| \leq \frac{C\|F\|}{\left(1-|\mathbf{z}|^{2}\right)^{\alpha+\beta}\left(1-\left|\varphi_{\mathbf{z}}(\mathbf{w})\right|^{2}\right)^{\beta} \omega(1-|\mathbf{w}|)} .
$$

This shows that $g \in \mathcal{B}_{\omega}^{\alpha, \beta}\left(\mathbb{B}_{n}\right)$ and completes the proof of the theorem.

Lemma 2.2 If $n>1, \alpha+\beta>\frac{1}{2}$, then $f \in \mathcal{B}_{\omega}^{\alpha, \beta}\left(\mathbb{B}_{n}\right)$ if and only if the function

$$
\frac{\left(1-|\mathbf{z}|^{2}\right)^{\alpha+\beta-1}}{\left(1-\left|\varphi_{\mathbf{w}}(\mathbf{z})\right|^{2}\right)^{\beta} \omega(1-|\mathbf{z}|)}|\widetilde{\nabla} f(\mathbf{z})|
$$

is bounded in $\mathbb{B}_{n}$.

Proof Recall from Lemma 2.14 in [5] that

$$
\left(1-|\mathbf{z}|^{2}\right)|\nabla f(\mathbf{z})| \leq|\widetilde{\nabla} f(\mathbf{z})|, \quad \mathbf{z} \in \mathbb{B}_{n} .
$$

So, the boundedness of

$$
\frac{\left(1-|\mathbf{z}|^{2}\right)^{\alpha+\beta-1}}{\left(1-\left|\varphi_{\mathbf{w}}(\mathbf{z})\right|^{2}\right)^{\beta} \omega(1-|\mathbf{z}|)}|\widetilde{\nabla} f(\mathbf{z})|
$$

implies that of

$$
\frac{\left(1-|\mathbf{z}|^{2}\right)^{\alpha+\beta}}{\left(1-\left|\varphi_{\mathbf{w}}(\mathbf{z})\right|^{2}\right)^{\beta} \omega(1-|\mathbf{z}|)}|\nabla f(\mathbf{z})|
$$


On the other hand, if $f \in \mathcal{B}_{\omega}^{\alpha, \beta}\left(\mathbb{B}_{n}\right)$, then by Theorem 2.1,

$$
f(\mathbf{z})=\left(1-\left|\varphi_{\mathbf{w}}(\mathbf{z})\right|^{2}\right)^{\beta} \omega(1-|\mathbf{z}|) \int_{\mathbb{B}_{n}} \frac{g(\mathbf{w}) d \nu(\mathbf{w})}{(1-\langle\mathbf{z}, \mathbf{w}\rangle)^{n+\alpha+\beta}}, \quad \mathbf{z} \in \mathbb{B}_{n},
$$

where $g$ is a function in $L^{\infty}\left(\mathbb{B}_{n}\right)$. Now we let $f(\mathbf{z})=h(\mathbf{z}) u(\mathbf{z})$, where $h(\mathbf{z})=\left(1-\left|\varphi_{\mathbf{w}}(\mathbf{z})\right|^{2}\right)^{\beta} \times$ $\omega(1-|\mathbf{z}|)$ and

$$
u(\mathbf{z})=\int_{\mathbb{B}_{n}} \frac{g(\mathbf{w}) d \nu(\mathbf{w})}{(1-\langle\mathbf{z}, \mathbf{w}\rangle)^{n+\alpha+\beta}} .
$$

An application of Lemma 1.1 gives

$$
|\widetilde{\nabla} u(\mathbf{z})| \leq|n+\alpha+\beta| \sqrt{2}\left(1-|\mathbf{z}|^{2}\right)^{\frac{1}{2}} \int_{\mathbb{B}_{n}} \frac{g(\mathbf{w}) d \nu(\mathbf{w})}{|1-\langle\mathbf{z}, \mathbf{w}\rangle|^{n+\alpha+\beta+\frac{1}{2}}}, \quad \forall \mathbf{z} \in \mathbb{B}_{n} .
$$

Since $g(\mathbf{z})$ is bounded, by Theorem 1.1 we have

$$
\int_{\mathbb{B}_{n}} \frac{g(\mathbf{w}) d v(\mathbf{w})}{|1-\langle\mathbf{z}, \mathbf{w}\rangle|^{n+\alpha+\beta+\frac{1}{2}}} \approx\left(1-|\mathbf{z}|^{2}\right)^{\frac{1}{2}-(\alpha+\beta)} .
$$

So,

$$
|\widetilde{\nabla} u(\mathbf{z})| \leq C\left(1-|\mathbf{z}|^{2}\right)^{1-(\alpha+\beta)} .
$$

It is easy to check that $\widetilde{\nabla} h(\mathbf{z})=\nabla\left(h \circ \varphi_{\mathbf{z}}\right)(0)=0$.

Using the product rule, we have

$$
|\widetilde{\nabla} f(\mathbf{z})| \leq|\widetilde{\nabla} h(\mathbf{z})||u(\mathbf{z})|+|h(\mathbf{z})||\widetilde{\nabla} u(\mathbf{z})| \leq|\widetilde{\nabla} h(\mathbf{z})||u(\mathbf{z})|
$$

and we have

$$
\begin{aligned}
& |\widetilde{\nabla} f(\mathbf{z})| \leq|n+\alpha+\beta| \sqrt{2}\left(1-|\mathbf{z}|^{2}\right)^{\frac{1}{2}}\left(1-\left|\varphi_{\mathbf{a}}(\mathbf{z})\right|^{2}\right)^{\beta} \int_{\mathbb{B}_{n}} \frac{g(\mathbf{w}) d \nu(\mathbf{w})}{|1-\langle\mathbf{z}, \mathbf{w}\rangle|^{n+\alpha+\beta+\frac{1}{2}}} \\
& \quad \text { for all } \mathbf{z} \in \mathbb{B}_{n} .
\end{aligned}
$$

Hence,

$$
\frac{\left(1-|\mathbf{z}|^{2}\right)^{\alpha+\beta-1}}{\left(1-\left|\varphi_{\mathbf{w}}(\mathbf{z})\right|^{2}\right)^{\beta} \omega(1-|\mathbf{z}|)}|\widetilde{\nabla} f(\mathbf{z})|
$$

is bounded in $\mathbb{B}_{n}$. This completes the proof.

Lemma 2.3 Let $0<\alpha+\beta \leq 2$. Let $\lambda$ be any real number satisfying the following properties:

- $0 \leq \lambda \leq \alpha+\beta$ if $0<\alpha+\beta<1$

- $0<\lambda<1$ if $\alpha+\beta=1$;

- $\alpha+\beta-1 \leq \lambda \leq 1$ if $1<\alpha+\beta \leq 2$.

Then, for all $\mathbf{z}, \mathbf{w} \in \mathbb{B}_{n}$ a holomorphic function $f \in \mathcal{B}_{\omega}^{\alpha, \beta}\left(\mathbb{B}_{n}\right)$ if and only if

$$
\sup _{\mathbf{z} \neq \mathbf{w}} \frac{\left(1-|\mathbf{z}|^{2}\right)^{\lambda}(1-|\mathbf{w}|)^{\alpha+\beta-\lambda}}{\left(1-\left|\varphi_{\mathbf{w}}(\mathbf{z})\right|^{2}\right)^{\beta} \omega(1-|\mathbf{z}|)} \frac{|f(\mathbf{z})-f(\mathbf{w})|}{|\mathbf{z}-\mathbf{w}|}<\infty .
$$


Proof Let $f \in \mathcal{B}_{\omega}^{\alpha, \beta}\left(\mathbb{B}_{n}\right)$. By a similar proof to the one for Theorem 3.1 in [20], we have

$$
|f(\mathbf{z})-f(\mathbf{w})|=\sqrt{n}|\mathbf{z}-\mathbf{w}| \int_{0}^{1}|\nabla f(t \mathbf{z}-(1-t) \mathbf{w})| d t
$$

for any $\mathbf{z}, \mathbf{w} \in \mathbb{B}_{n}$ with $\mathbf{z} \neq \mathbf{w}$. We know that

$$
\|f\|_{\mathcal{B}_{\omega}^{\alpha, \beta}\left(\mathbb{B}_{n}\right)} \approx \sup _{\mathbf{w}, \mathbf{z} \in \mathbb{B}_{n}} \frac{\left(1-|\mathbf{z}|^{2}\right)^{\alpha+\beta}}{\left(1-\left|\varphi_{\mathbf{w}}(\mathbf{z})\right|^{2}\right)^{\beta} \omega(1-|\mathbf{z}|)}|\nabla f(\mathbf{z})|
$$

Thus, there is a constant $C>0$ such that

$$
\frac{|f(\mathbf{z})-f(\mathbf{w})|}{|\mathbf{z}-\mathbf{w}|} \leq C\|f\|_{\mathcal{B}_{\omega}^{\alpha, \beta}\left(\mathbb{B}_{n}\right)} \int_{0}^{1} \frac{\left(1-\left|\varphi_{\mathbf{a}}(t \mathbf{z}-(1-t) \mathbf{a})\right|^{2}\right)^{\beta} \omega(1-|t \mathbf{z}-(1-t) \mathbf{w}|)}{\left(1-|t \mathbf{z}-(1-t) \mathbf{w}|^{2}\right)^{\alpha+\beta}} d t .
$$

Since $(1-|\mathbf{z}|) \leq|1-\langle\mathbf{w}, \mathbf{z}\rangle|$ and

$$
1-|t \mathbf{z}+(1-t) \mathbf{w}|^{2} \geq 1-|t \mathbf{z}+(1-t) \mathbf{w}| \geq 1-|\mathbf{w}|+(|\mathbf{w}|-|\mathbf{z}|) t
$$

we get

$$
\begin{aligned}
\left(1-\left|\varphi_{\mathbf{w}}(t \mathbf{z}-(1-t) \mathbf{w})\right|^{2}\right)^{\beta} & =\frac{\left(1-|\mathbf{w}|^{2}\right)^{\beta}\left(1-|t \mathbf{z}-(1-t) \mathbf{w}|^{2}\right)^{\beta}}{|1-\langle t \mathbf{z}-(1-t) \mathbf{w}, \mathbf{w}\rangle|^{2 \beta}} \\
& \leq \frac{\left(1-|\mathbf{w}|^{2}\right)^{\beta}\left(1-|t \mathbf{z}-(1-t) \mathbf{w}|^{2}\right)^{\beta}}{(1-|\mathbf{w}|)^{2 \beta}} \\
& \leq \frac{(1+|\mathbf{w}|)^{\beta}\left(1-|t \mathbf{z}-(1-t) \mathbf{w}|^{2}\right)^{\beta}}{(1-|\mathbf{w}|)^{\beta}} \\
& \leq \frac{\left(1-|t \mathbf{z}-(1-t) \mathbf{w}|^{2}\right)^{\beta}}{(1-|\mathbf{w}|)^{\beta}}
\end{aligned}
$$

Thus

$$
\frac{|f(\mathbf{z})-f(\mathbf{w})|}{|\mathbf{z}-\mathbf{w}|} \leq C\|f\|_{\mathcal{B}_{\omega}^{\alpha, \beta}\left(\mathbb{B}_{n}\right)} \int_{0}^{1} \frac{1}{(1-|\mathbf{w}|+(|\mathbf{w}|-|\mathbf{z}|) t)^{\alpha}(1-|\mathbf{w}|)^{\beta}} d t .
$$

If $|\mathbf{z}|=|\mathbf{a}|$, then

$$
\frac{|f(\mathbf{z})-f(\mathbf{w})|}{|\mathbf{z}-\mathbf{w}|} \leq C\|f\|_{\mathcal{B}_{\omega}^{\alpha, \beta}\left(\mathbb{B}_{n}\right)} \int_{0}^{1} \frac{1}{(1-|\mathbf{w}|)^{\alpha+\beta}} d t \leq \frac{C\|f\|_{\mathcal{B}_{\omega}^{\alpha, \beta}\left(\mathbb{B}_{n}\right)}}{\left(1-|\mathbf{z}|^{2}\right)^{\lambda}\left(1-|\mathbf{w}|^{2}\right)^{\alpha+\beta-\lambda}} .
$$

Now suppose $|\mathbf{z}| \neq|\mathbf{w}|$ as in [21], there is a constant $C>0$ such that this integral in (4) is dominated by

$$
\frac{C}{\left(1-|\mathbf{z}|^{2}\right)^{\lambda}\left(1-|\mathbf{w}|^{2}\right)^{\alpha+\beta-\lambda}} .
$$

Combining with (5), we get that whenever $\mathbf{z} \neq \mathbf{w}$,

$$
\frac{|f(\mathbf{z})-f(\mathbf{w})|}{|\mathbf{z}-\mathbf{w}|} \leq \frac{C\|f\|_{\mathcal{B}_{\omega}^{\alpha, \beta}\left(\mathbb{B}_{n}\right)}}{\left(1-|\mathbf{z}|^{2}\right)^{\lambda}\left(1-|\mathbf{w}|^{2}\right)^{\alpha+\beta-\lambda}} .
$$


This proves the necessity. The proof of the sufficiency condition is much akin to the corresponding one in [21], so the proof is omitted.

Proposition 2.1 Suppose $f \in \mathcal{B}_{\omega}^{\alpha, \beta}\left(\mathbb{B}_{n}\right)$ and $1 \leq \alpha+\beta \leq 2$. Let $\lambda$ be any real number satisfying:

- $0<\lambda<1$ if $\alpha+\beta=1$;

- $\alpha+\beta-1 \leq \lambda \leq 1$ if $1<\alpha+\beta \leq 2$.

Then

$$
\begin{aligned}
& \sup _{\mathbf{z}, \mathbf{w} \in \mathbb{B}_{n}} \frac{\left(1-|\mathbf{z}|^{2}\right)^{2 \alpha+2 \beta-\lambda-1}\left(1-|\mathbf{w}|^{2}\right)^{\alpha+\beta-\lambda}|f(\mathbf{z})-f(\mathbf{w})|}{\left(1-\left|\varphi_{\mathbf{w}}(\mathbf{z})\right|^{2}\right)^{\beta} \omega(1-|\mathbf{z}|)|1-\langle\mathbf{w}, \mathbf{z}\rangle|^{2(\alpha+\beta)-(2 \lambda+1)}\left|\mathbf{z}-P_{\mathbf{z}}(\mathbf{w})-S_{\mathbf{z}} Q_{\mathbf{z}}(\mathbf{w})\right|} \\
& \quad \leq C\|f\|_{\mathcal{B}_{\omega}^{\alpha, \beta}\left(\mathbb{B}_{n}\right)^{.}}
\end{aligned}
$$

Proof Let $\mathbf{z}=0$ in (3), then we have

$$
\left(1-|\mathbf{w}|^{2}\right)^{\alpha+\beta-\lambda} \frac{|f(\mathbf{0})-f(\mathbf{w})|}{|\mathbf{w}|} \leq C b_{\alpha, \beta ; \omega}(f)\left(\mathbb{B}_{n}\right), \quad \mathbf{w} \in \mathbb{B}_{n} \backslash\{\mathbf{0}\} .
$$

Now, replacing $f$ by $f \circ \varphi_{\mathbf{w}}$, we get

$$
\left(1-|\mathbf{u}|^{2}\right)^{\alpha+\beta-\lambda} \frac{\left|f \circ \varphi_{\mathbf{w}}(\mathbf{0})-f \circ \varphi_{\mathbf{w}}(\mathbf{u})\right|}{|\mathbf{u}|} \leq C b_{\alpha, \beta ; \omega}\left(f \circ \varphi_{\mathbf{w}}\right)\left(\mathbb{B}_{n}\right), \quad \mathbf{u} \in \mathbb{B}_{n} \backslash\{\mathbf{0}\}
$$

Since

$$
\left|\widetilde{\nabla}\left(f \circ \varphi_{\mathbf{w}}\right)(\mathbf{z})\right|=\left|\widetilde{\nabla} f\left(\varphi_{\mathbf{w}}(\mathbf{z})\right)\right|,
$$

by Lemma 2.2, we obtain that

$$
\begin{aligned}
b_{\alpha, \beta ; \omega}\left(f \circ \varphi_{\mathbf{w}}\right)\left(\mathbb{B}_{n}\right) & \approx \sup _{\mathbf{z}, \mathbf{w} \in \mathbb{B}_{n}} \frac{\left(1-|\mathbf{w}|^{2}\right)^{\alpha+\beta-1}}{\left(1-\left|\varphi_{\mathbf{w}}(\mathbf{z})\right|^{2}\right)^{\beta} \omega(1-|\mathbf{z}|)}\left|\widetilde{\nabla}\left(f \circ \varphi_{\mathbf{w}}\right)(\mathbf{z})\right| \\
& =\sup _{\mathbf{z}, \mathbf{w} \in \mathbb{B}_{n}} \frac{\left(1-|\mathbf{w}|^{2}\right)^{\alpha+\beta-1}}{\left(1-\left|\varphi_{\mathbf{w}}(\mathbf{z})\right|^{2}\right)^{\beta} \omega(1-|\mathbf{z}|)}\left|\widetilde{\nabla} f\left(\varphi_{\mathbf{w}}(\mathbf{z})\right)\right| \\
& =\sup _{\mathbf{z}, \mathbf{w} \in \mathbb{B}_{n}} \frac{\left(1-|\mathbf{w}|^{2}\right)^{\alpha+\beta-1}\left(1-\left|\varphi_{\mathbf{w}}(\mathbf{z})\right|^{2}\right)^{\alpha+\beta-1}}{\left(1-\left|\varphi_{\mathbf{w}}(\mathbf{z})\right|^{2}\right)^{\alpha+\beta-1}\left(1-\left|\varphi_{\mathbf{w}}(\mathbf{z})\right|^{2}\right)^{\beta} \omega(1-|\mathbf{z}|)}\left|\widetilde{\nabla} f\left(\varphi_{\mathbf{w}}(\mathbf{z})\right)\right| .
\end{aligned}
$$

Then

$$
\begin{aligned}
b_{\alpha, \beta ; \omega}\left(f \circ \varphi_{\mathbf{z}}\right)\left(\mathbb{B}_{n}\right) & \leq C\|f\|_{\mathcal{B}_{\omega}^{\alpha, \beta}\left(\mathbb{B}_{n}\right)} \frac{\left(1-|\mathbf{w}|^{2}\right)^{\alpha+\beta-1}}{\left(1-\left|\varphi_{\mathbf{z}}(\mathbf{w})\right|^{2}\right)^{\alpha+\beta-1} \omega(1-|\mathbf{w}|)} \\
& \leq \frac{C\|f\|_{\mathcal{B}_{\omega}^{\alpha, \beta}\left(\mathbb{B}_{n}\right)}}{\left(1-|\mathbf{z}|^{2}\right)^{\alpha+\beta-1}} .
\end{aligned}
$$

Letting $u=\varphi_{\mathbf{w}}(\mathbf{z})$ and $\mathbf{w} \neq \mathbf{z}$ in (7), we obtain

$$
\frac{|f(\mathbf{z})-f(\mathbf{w})|}{\left|\varphi_{\mathbf{w}}(\mathbf{z})\right| \omega(1-|\mathbf{z}|)\left(1-\left|\varphi_{\mathbf{w}}(\mathbf{z})\right|^{2}\right)^{\alpha+\beta-\lambda}} \leq \frac{C\|f\|_{\mathcal{B}_{\omega}^{\alpha, \beta}\left(\mathbb{B}_{n}\right)}}{\left(1-|\mathbf{z}|^{2}\right)^{\alpha+\beta-1}} .
$$


Since

$$
1-\left|\varphi_{\mathbf{z}}(\mathbf{w})\right|^{2}=\frac{\left(1-|\mathbf{z}|^{2}\right)\left(1-|\mathbf{w}|^{2}\right)}{|1-\langle\mathbf{w}, \mathbf{z}\rangle|^{2}}
$$

and

$$
\varphi_{\mathbf{z}}(\mathbf{w})=\frac{\mathbf{z}-P_{\mathbf{z}}(\mathbf{w})-S_{\mathbf{z}} Q_{\mathbf{z}}(\mathbf{w})}{1-\langle\mathbf{w}, \mathbf{z}\rangle} .
$$

Consequently,

$$
\begin{aligned}
& \frac{\left(1-|\mathbf{z}|^{2}\right)^{2 \alpha+2 \beta-\lambda-1}\left(1-|\mathbf{w}|^{2}\right)^{\alpha+\beta-\lambda}|f(\mathbf{z})-f(\mathbf{w})|}{\omega(1-|\mathbf{z}|)\left(1-\left|\varphi_{\mathbf{z}}(\mathbf{w})\right|^{2}\right)^{\alpha+\beta-\lambda}|1-\langle\mathbf{w}, \mathbf{z}\rangle|^{2(\alpha+\beta)-(2 \lambda+1)}\left|\mathbf{z}-P_{\mathbf{z}}(\mathbf{w})-S_{\mathbf{z}} Q_{\mathbf{z}}(\mathbf{w})\right|} \\
& \quad \leq C\|f\|_{\mathcal{B}_{\omega}^{\alpha, \beta}\left(\mathbb{B}_{n}\right)} .
\end{aligned}
$$

\section{Boundedness of general Toeplitz operators}

In this section, we study the boundedness of general Toeplitz operators acting on the weighted Bloch-type spaces $\mathcal{B}_{\omega}^{\alpha, \beta}\left(\mathbb{B}_{n}\right)$ in the unit ball of $\mathbb{C}^{n}$.

Theorem 3.1 Let $\mu$ be a positive Borel measure on $\mathbb{B}_{n}$. Then we have

(1) if $\alpha+\beta=1$, then $T_{\mu}^{\alpha, \beta ; \omega}$ is bounded on $\mathcal{B}_{\omega}^{\alpha, \beta}\left(\mathbb{B}_{n}\right)$ if and only if $P_{\alpha+\beta-1 ; \omega}(\mu) \in \mathcal{L} \mathcal{B}_{\omega}\left(\mathbb{B}_{n}\right)$ and $\mu$ is a Carleson measure;

(2) if $\alpha=\beta=1$, then $T_{\mu}^{\alpha, \beta ; \omega}$ is bounded on $\mathcal{B}_{\omega}^{\alpha, \beta}\left(\mathbb{B}_{n}\right)$ if and only if $P_{\alpha+\beta-1 ; \omega}(\mu) \in \mathcal{B}_{\omega}\left(\mathbb{B}_{n}\right) \cap \mathcal{L B}_{\omega}^{2}\left(\mathbb{B}_{n}\right)$ and $\mu$ is a Carleson measure;

(3) if $\alpha>1, \beta>1$, then $T_{\mu}^{\alpha, \beta ; \omega}$ is bounded on $\mathcal{B}_{\omega}^{\alpha, \beta}\left(\mathbb{B}_{n}\right)$ if and only if $P_{\alpha+\beta-1 ; \omega}(\mu) \in \mathcal{B}_{\omega}^{\alpha, \beta}\left(\mathbb{B}_{n}\right)$ and $\mu$ is a Carleson measure.

Proof Since the Banach dual of $A^{1}\left(\mathbb{B}_{n}\right)$ is $\mathcal{B}_{\omega}^{\alpha, \beta}\left(\mathbb{B}_{n}\right)$ under the pairing (2), to prove the boundedness of $T_{\mu}^{\alpha, \beta ; \omega}$, it suffices to show that

$$
\left|\left\langle f, T_{\mu}^{\alpha, \beta ; \omega}(g)\right\rangle_{\alpha}\right| \leq C\|f\|_{A^{1}\left(\mathbb{B}_{n}\right)}\|g\|_{\mathcal{B}_{\omega}^{\alpha, \beta}\left(\mathbb{B}_{n}\right)}
$$

for all $f \in A^{1}\left(\mathbb{B}_{n}\right)$ and $g \in \mathcal{B}_{\omega}^{\alpha, \beta}\left(\mathbb{B}_{n}\right)$, where $C$ is a positive constant that does not depend on $f$ or $g$.

For $s=\alpha+\beta-1$, by Fubini's theorem, we get

$$
\begin{aligned}
\left\langle f, T_{\mu}^{\alpha, \beta ; \omega} g\right\rangle_{s} & =\int_{\mathbb{B}_{n}} f(\mathbf{z}) \overline{T_{\mu}^{\alpha, \beta ; \omega} g(\mathbf{z})} d \nu_{s}(\mathbf{z}) \\
& =c_{\alpha+\beta-1} \int_{\mathbb{B}_{n}} f(\mathbf{z}) \overline{g(\mathbf{z})}\left(1-|\mathbf{z}|^{2}\right)^{\alpha+\beta-1} d \mu(\mathbf{z}) .
\end{aligned}
$$

Using the operator $P_{\alpha+\beta ; \omega}$, we have

$$
\begin{aligned}
\left\langle f, T_{\mu}^{\alpha, \beta ; \omega} g\right\rangle_{s}= & c_{\alpha+\beta-1} \int_{\mathbb{B}_{n}}\left(I_{\mathbf{z}, \mathbf{w} ; \omega}-P_{\alpha+\beta ; \omega}\right)(f \bar{g})(\mathbf{z})\left(1-|\mathbf{z}|^{2}\right)^{\alpha+\beta-1} d \mu(\mathbf{z}) \\
& +c_{\alpha+\beta-1} \int_{\mathbb{B}_{n}} P_{\alpha+\beta ; \omega}(f \bar{g})(\mathbf{z})\left(1-|\mathbf{z}|^{2}\right)^{\alpha+\beta-1} d \mu(\mathbf{z}) \\
= & I_{1}+I_{2}
\end{aligned}
$$


where $I_{\mathbf{z}, \mathbf{w} ; \omega}=\frac{I}{\left(1-\left|\varphi_{\mathbf{Z}}(\mathbf{w})\right|^{2}\right)^{\beta} \omega(1-|\mathbf{w}|)}$, and $I$ is the identity operator. Also,

$$
\begin{aligned}
\left(I_{\mathbf{z}, \mathbf{w} ; \omega}-P_{\alpha+\beta ; \omega}\right)(f \bar{g})(\mathbf{z}) \\
=\frac{f(\mathbf{z}) \overline{g(\mathbf{z})}}{\left(1-\left|\varphi_{\mathbf{z}}(\mathbf{w})\right|^{2}\right)^{\beta} \omega(1-|\mathbf{w}|)} \\
\quad-\frac{c_{\alpha+\beta}}{\left(1-\left|\varphi_{\mathbf{z}}(\mathbf{w})\right|^{2}\right)^{\beta} \omega(1-|\mathbf{w}|)} \int_{\mathbb{B}_{n}} \frac{f(\mathbf{w}) \overline{g(\mathbf{w})}\left(1-|\mathbf{w}|^{2}\right)^{\alpha+\beta}}{(1-\langle\mathbf{z}, \mathbf{w}\rangle)^{n+\alpha+\beta+1}} d \nu(\mathbf{w}) \\
=\frac{c_{\alpha+\beta}}{\left(1-\left|\varphi_{\mathbf{z}}(\mathbf{w})\right|^{2}\right)^{\beta} \omega(1-|\mathbf{w}|)} \\
\quad \times \int_{\mathbb{B}_{n}} \frac{(\overline{g(\mathbf{z})}-\overline{g(\mathbf{w})}) f(\mathbf{w})\left(1-|\mathbf{w}|^{2}\right)^{\alpha+\beta}}{(1-\langle\mathbf{z}, \mathbf{w}\rangle)^{n+\alpha+\beta+1}\left(1-\left|\varphi_{\mathbf{z}}(\mathbf{w})\right|^{2}\right)^{\beta} \omega(1-|\mathbf{w}|)} d \nu(\mathbf{z}) .
\end{aligned}
$$

By Proposition 2.1, we have

$$
\begin{aligned}
& \left|I_{1}\right|=c_{\alpha+\beta-1}\left|\int_{\mathbb{B}_{n}}\left(I_{\mathbf{z}, \mathbf{w} ; \omega}-P_{\alpha+\beta ; \omega}\right)(f \bar{g})(\mathbf{z})\left(1-|\mathbf{z}|^{2}\right)^{\alpha+\beta-1} d \mu(\mathbf{z})\right|
\end{aligned}
$$

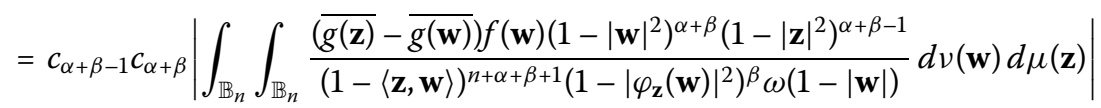

$$
\begin{aligned}
& =c_{\alpha+\beta-1} c_{\alpha+\beta} \mid \int_{\mathbb{B}_{n}} f(\mathbf{w})\left(1-|\mathbf{w}|^{2}\right)^{\alpha+\beta} \\
& \times \int_{\mathbb{B}_{n}} \frac{(\overline{g(\mathbf{z})}-\overline{g(\mathbf{w})})\left(1-|\mathbf{z}|^{2}\right)^{\alpha+\beta-1}}{(1-\langle\mathbf{z}, \mathbf{w}\rangle)^{n+\alpha+\beta+1}\left(1-\left|\varphi_{\mathbf{z}}(\mathbf{w})\right|^{2}\right)^{\beta} \omega(1-|\mathbf{w}|)} d \mu(\mathbf{z}) d v(\mathbf{w}) \mid \\
& \leq c_{\alpha+\beta-1} c_{\alpha+\beta} \int_{\mathbb{B}_{n}}|f(\mathbf{w})|\left(1-|\mathbf{w}|^{2}\right)^{\lambda} \\
& \times \int_{\mathbb{B}_{n}} \frac{\left(1-|\mathbf{z}|^{2}\right)^{2(\alpha+\beta)-\lambda-1}\left(1-|\mathbf{w}|^{2}\right)^{\alpha+\beta-\lambda}|f(\mathbf{z})-f(\mathbf{w})|}{|1-\langle\mathbf{w}, \mathbf{z}\rangle|^{2(\alpha+\beta)-(2 \lambda+1)}\left|\mathbf{z}-P_{\mathbf{z}}(\mathbf{w})-S_{\mathbf{z}} Q_{\mathbf{z}}(\mathbf{w})\right|} \\
& \times \frac{\left|\mathbf{z}-P_{\mathbf{z}}(\mathbf{w})-S_{\mathbf{z}} Q_{\mathbf{z}}(\mathbf{w})\right|\left(1-|\mathbf{z}|^{2}\right)^{\lambda-(\alpha+\beta)}}{|1-\langle\mathbf{z}, \mathbf{w}\rangle|^{n-(\alpha+\beta)+2 \lambda+2}\left(1-\left|\varphi_{\mathbf{z}}(\mathbf{w})\right|^{2}\right)^{\beta} \omega(1-|\mathbf{w}|)} d \mu(\mathbf{z}) d \nu(\mathbf{w}) \\
& \leq C \int_{\mathbb{B}_{n}}\|g\|_{\mathcal{B}_{\omega}^{\alpha, \beta}\left(\mathbb{B}_{n}\right)}|f(\mathbf{w})|\left(1-|\mathbf{w}|^{2}\right)^{\lambda} \int_{\mathbb{B}_{n}} \frac{\left(1-|\mathbf{z}|^{2}\right)^{\lambda-(\alpha+\beta)}}{|1-\langle\mathbf{z}, \mathbf{w}\rangle|^{n-(\alpha+\beta)+2 \lambda+1}} d \mu(\mathbf{z}) d v(\mathbf{w}) .
\end{aligned}
$$

Since $\mu$ is a Carleson measure, taking $\lambda-(\alpha+\beta)>-1$, then as in [9] or in [22, Proposition 1.4.10], for fixed $r>0$, we get

$$
\begin{aligned}
& \int_{\mathbb{B}_{n}} \frac{\left(1-|\mathbf{z}|^{2}\right)^{\lambda-(\alpha+\beta)}}{|1-\langle\mathbf{z}, \mathbf{w}\rangle|^{n-(\alpha+\beta)+2 \lambda+1}} d \mu(\mathbf{z}) \\
& \quad \leq \sum_{j=1}^{\infty} \frac{\mu\left(B\left(\mathbf{z}^{(j)}, r\right)\right)}{v\left(B\left(\mathbf{z}^{(j)}, r\right)\right)} \int_{B\left(\mathbf{z}^{(j)}, r\right)} \frac{\left(1-|\mathbf{z}|^{2}\right)^{\lambda-(\alpha+\beta)}}{|1-\langle\mathbf{z}, \mathbf{w}\rangle|^{n-(\alpha+\beta)+2 \lambda+1}} d v(\mathbf{z}) \leq C .
\end{aligned}
$$

Therefore,

$$
\left|I_{1}\right| \leq C\|f\|_{A^{1}\left(\mathbb{B}_{n}\right)}\|g\|_{\mathcal{B}_{\omega}^{\alpha, \beta}\left(\mathbb{B}_{n}\right)} \cdot
$$


Next considering $I_{2}$, we have

$$
\begin{aligned}
\left|I_{2}\right|= & c_{\alpha+\beta-1}\left|\int_{\mathbb{B}_{n}} P_{\alpha+\beta ; \omega}(f \bar{g})(\mathbf{z})\left(1-|\mathbf{z}|^{2}\right)^{\alpha+\beta-1} d \mu(\mathbf{z})\right| \\
= & c_{\alpha+\beta-1} c_{\alpha+\beta}\left|\int_{\mathbb{B}_{n}} \int_{\mathbb{B}_{n}} \frac{f(\mathbf{w}) \overline{g(\mathbf{w})}\left(1-|\mathbf{w}|^{2}\right)^{\alpha+\beta}\left(1-|\mathbf{z}|^{2}\right)^{\alpha+\beta-1}}{(1-\langle\mathbf{z}, \mathbf{w}\rangle)^{n+\alpha+\beta+1}\left(1-\left|\varphi_{\mathbf{z}}(\mathbf{w})\right|^{2}\right)^{\beta} \omega(1-|\mathbf{w}|)} d \nu(\mathbf{w}) d \mu(\mathbf{z})\right| \\
\leq & c_{\alpha+\beta} \int_{\mathbb{B}_{n}}|f(\mathbf{w})|\left(1-|\mathbf{w}|^{2}\right)^{\alpha+\beta}|g(\mathbf{w})| \\
& \times\left(\frac{c_{\alpha+\beta-1}}{\left(1-\left|\varphi_{\mathbf{z}}(\mathbf{w})\right|^{2}\right)^{\beta} \omega(1-|\mathbf{w}|)} \int_{\mathbb{B}_{n}} \frac{\left(1-|\mathbf{z}|^{2}\right)^{\alpha+\beta-1} d \mu(\mathbf{z})}{|1-\langle\mathbf{z}, \mathbf{w}\rangle|^{n+\alpha+\beta+1}}\right) d \nu(\mathbf{w}) \\
\leq & C \int_{\mathbb{B}_{n}}\|f\|_{A^{1}\left(\mathbb{B}_{n}\right)}\left(1-|\mathbf{w}|^{2}\right)^{\alpha+\beta}|g(\mathbf{w})| Q_{\mu}^{\alpha, \beta ; \omega}(\mathbf{w}) d \nu(\mathbf{w}),
\end{aligned}
$$

where

$$
Q_{\mu}^{\alpha, \beta ; \omega}(\mathbf{w})=\frac{c_{\alpha+\beta-1}}{\left(1-\left|\varphi_{\mathbf{z}}(\mathbf{w})\right|^{2}\right)^{\beta} \omega(1-|\mathbf{w}|)} \int_{\mathbb{B}_{n}} \frac{\left(1-|\mathbf{z}|^{2}\right)^{\alpha+\beta-1} d \mu(\mathbf{z})}{|1-\langle\mathbf{z}, \mathbf{w}\rangle|^{n+\alpha+\beta+1}}
$$

As in [9], by simple calculation, we have

$$
Q_{\mu}^{\alpha, \beta ; \omega}(\mathbf{w})=P_{\alpha+\beta-1 ; \omega}(\mu)(\mathbf{w})+\frac{1}{n+\alpha+\beta} \Re P_{\alpha+\beta-1 ; \omega}(\mu)(\mathbf{w}) .
$$

It is easy to see that

(1) if $\alpha+\beta=1$ and $P_{\alpha+\alpha-1 ; \omega}(\mu) \in \mathcal{L} \mathcal{B}_{\omega}^{\alpha, \beta}\left(\mathbb{B}_{n}\right)$, then

$$
\left(1-|\mathbf{w}|^{2}\right) Q_{\mu}^{\alpha, \beta ; \omega}(\mathbf{w})\left(\ln \frac{2}{1-|\mathbf{w}|^{2}}\right) \in L^{\infty}\left(\mathbb{B}_{n}\right) ;
$$

(2) if $\alpha=\beta=1, P_{\alpha+\alpha-1 ; \omega}(\mu) \in \mathcal{B}_{\omega}\left(\mathbb{B}_{n}\right) \cap \mathcal{L B}-\omega^{2}\left(\mathbb{B}_{n}\right)$, then

$$
\left(1-|\mathbf{w}|^{2}\right) Q_{\mu}^{\alpha, \beta ; \omega}(\mathbf{w}) \in L^{\infty}\left(\mathbb{B}_{n}\right)
$$

and

$$
\left(1-|\mathbf{w}|^{2}\right)^{2} Q_{\mu}^{\alpha, \beta ; \omega}(\mathbf{w})\left(\ln \frac{2}{1-|\mathbf{w}|^{2}}\right) \in L^{\infty}\left(\mathbb{B}_{n}\right)
$$

(3) if $\alpha>1, \beta>1$, and $P_{\alpha+\alpha-1 ; \omega}(\mu) \in \mathcal{B}-\omega^{\alpha, \beta}\left(\mathbb{B}_{n}\right)$, then

$$
\left(1-|\mathbf{w}|^{2}\right)^{\alpha+\beta} Q_{\mu}^{\alpha, \beta ; \omega}(\mathbf{w}) \in L^{\infty}\left(\mathbb{B}_{n}\right) .
$$

This implies that $\left|I_{2}\right| \leq C\|f\|_{A^{1}\left(\mathbb{B}_{n}\right)}\|g\|_{\mathcal{B}_{\omega}^{\alpha, \beta}\left(\mathbb{B}_{n}\right)}$. Hence, $T_{\mu}^{\alpha, \beta ; \omega}$ is a bounded operator on $\mathcal{B}_{\omega}^{\alpha, \beta}\left(\mathbb{B}_{n}\right)$ with $\alpha>0, \beta \geq 0$.

Conversely, suppose that $T_{\mu}^{\alpha, \beta ; \omega}$ is a bounded operator on $\mathcal{B}_{\omega}^{\alpha, \beta}\left(\mathbb{B}_{n}\right)$. Take

$$
f_{\mathbf{w}}(\mathbf{z})=\frac{\left(1-|\mathbf{w}|^{2}\right)^{t}}{(1-\langle\mathbf{z}, \mathbf{w}\rangle)^{n+t+1}} \quad \text { for } t>0 .
$$


It is clear that $\left\|f_{\mathrm{w}}\right\|_{A^{1}\left(\mathbb{B}_{n}\right)} \leq C$. On the other hand, take

$$
g_{\mathbf{w}}(\mathbf{z})=\frac{\left(1-|\mathbf{w}|^{2}\right)^{n+2+t-(\alpha+\beta)}}{(1-\langle\mathbf{z}, \mathbf{w}\rangle)^{n+t+1}} ; \quad \varphi_{\mathbf{w}}(\mathbf{z}) \equiv 1 \quad \text { and } \quad \omega(1-|\mathbf{z}|) \equiv 1 \quad \text { for } t>0 .
$$

Then, we have $\left\|g_{\mathbf{w}}\right\|_{\mathcal{B}_{\omega}^{\alpha, \beta}\left(\mathbb{B}_{n}\right)} \leq C$. Therefore

$$
\begin{aligned}
& \left|\left\langle f, T_{\mu}^{\alpha} g\right\rangle_{s}\right|=c_{\alpha+\beta-1}\left(1-|\mathbf{w}|^{2}\right)^{n+2+2 t-(\alpha+\beta)} \int_{\mathbb{B}_{n}} \frac{\left(1-|\mathbf{z}|^{2}\right)^{\alpha+\beta-1} d \mu(\mathbf{z})}{|1-\langle\mathbf{z}, \mathbf{w}\rangle|^{2(n+t+1)}} \\
& \leq C\left\|T_{\mu}^{\alpha, \beta ; \omega}\right\|\left\|f_{\mathbf{w}}\right\|_{A^{1}\left(\mathbb{B}_{n}\right)}\left\|g_{\mathbf{w}}\right\|_{\mathcal{B}_{\omega}^{\alpha, \beta}\left(\mathbb{B}_{n}\right)} \leq C .
\end{aligned}
$$

Thus,

$$
\left(1-|\mathbf{w}|^{2}\right)^{n+2+2 t-(\alpha+\beta)} \int_{B(\mathbf{w}, r)} \frac{\left(1-|\mathbf{z}|^{2}\right)^{\alpha+\beta-1} d \mu(\mathbf{z})}{|1-\langle\mathbf{z}, \mathbf{w}\rangle|^{2(n+t+1)}} \leq C
$$

for every $\mathbf{w} \in \mathbb{B}_{n}$. This implies that

$$
\sup _{\mathbf{w} \in \mathbb{B}_{n}} \frac{\mu(B(\mathbf{w}, r))}{v(B(\mathbf{w}, r))}<\infty
$$

Hence $\mu$ is a Carleson measure on $\mathbb{B}_{n}$.

From the proof of the sufficient condition, we find that there exists a constant $C$ such that

$$
\begin{aligned}
\left|I_{2}\right| & =c_{\alpha+\beta}\left|\int_{\mathbb{B}_{n}} f(\mathbf{w})\left(1-|\mathbf{w}|^{2}\right)^{\alpha+\beta} \overline{g(\mathbf{w}) Q_{\mu}^{\alpha, \beta ; \omega}(\mathbf{w})} d \nu(\mathbf{w})\right| \\
& \leq C\|f\|_{A^{1}\left(\mathbb{B}_{n}\right)}\|g\|_{\mathcal{B}_{\omega}^{\alpha, \beta}\left(\mathbb{B}_{n}\right)} .
\end{aligned}
$$

This implies that

$$
\left|g(\mathbf{w}) Q_{\mu}^{\alpha, \beta ; \omega}(\mathbf{w})\right|\left(1-|\mathbf{w}|^{2}\right)^{\alpha+\beta} \leq C\|g\|_{\mathcal{B}_{\omega}^{\alpha, \beta}\left(\mathbb{B}_{n}\right)} .
$$

If $\alpha+\beta=1$, we have

$$
\left|g(\mathbf{w}) Q_{\mu}^{\alpha, \beta ; \omega}(\mathbf{w})\right|\left(1-|\mathbf{w}|^{2}\right) \leq C\|g\|_{\mathcal{B}_{\omega}^{\alpha, \beta}\left(\mathbb{B}_{n}\right)} .
$$

Take $g_{\mathbf{w}}(\mathbf{z})=\ln \frac{2}{1-\langle\mathbf{z}, \mathbf{w}\rangle} ; \varphi_{\mathbf{w}}(\mathbf{z}) \equiv 1$ and $\omega(1-|\mathbf{z}|) \equiv 1$. It is clear that $\left\|g_{\mathbf{w}}\right\|_{\mathcal{L B}_{\omega}\left(\mathbb{B}_{n}\right)} \leq C$. Taking $\mathbf{z}=\mathbf{w}$, then

$$
\left|Q_{\mu}^{\alpha, \beta ; \omega}(\mathbf{w})\right|\left(1-|\mathbf{w}|^{2}\right)\left(\ln \frac{2}{1-|\mathbf{w}|^{2}}\right) \leq C .
$$

From (8) we have $P_{\alpha+\beta-1}(\mu) \in \mathcal{L B}_{\omega}\left(\mathbb{B}_{n}\right)$. Let $\alpha=\beta=1$, we have

$$
\left|g(\mathbf{w}) Q_{\mu}^{\alpha, \beta ; \omega}(\mathbf{w})\right|\left(1-|\mathbf{w}|^{2}\right)^{2} \leq C\|g\|_{\mathcal{B}_{\omega}^{\alpha, \beta}\left(\mathbb{B}_{n}\right)} .
$$

Take $g_{\mathbf{w}}(\mathbf{z})=\frac{1}{1-\langle\mathbf{z}, \mathbf{w}\rangle}+\ln \frac{2}{1-\langle\mathbf{z}, \mathbf{w}\rangle} ; \varphi_{\mathbf{w}}(\mathbf{z}) \equiv 1$ and $\omega(1-|\mathbf{z}|) \equiv 1$. It is clear that

$$
\left\|g_{\mathbf{w}}\right\|_{\mathcal{B}_{\omega}\left(\mathbb{B}_{n}\right) \cap \mathcal{L} \mathcal{B}_{\omega}^{2}\left(\mathbb{B}_{n}\right)} \leq C
$$


Taking $\mathbf{z}=\mathbf{w}$, then

$$
\begin{aligned}
& \left|g(\mathbf{w}) Q_{\mu}^{\alpha, \beta ; \omega}(\mathbf{w})\right|\left(1-|\mathbf{w}|^{2}\right)^{2} \\
& \quad=\left(\frac{1}{1-|\mathbf{w}|^{2}}+\ln \frac{2}{1-|\mathbf{w}|^{2}}\right)\left|Q_{\mu}^{\alpha, \beta}(\mathbf{w})\right|\left(1-|\mathbf{w}|^{2}\right)^{2} \\
& \quad \leq\left|Q_{\mu}^{\alpha, \beta ; \omega}(\mathbf{w})\right|\left(1-|\mathbf{w}|^{2}\right)+\left|Q_{\mu}^{\alpha, \beta ; \omega}(\mathbf{w})\right|\left(1-|\mathbf{w}|^{2}\right)^{2}\left(\ln \frac{2}{1-|\mathbf{w}|^{2}}\right) \\
& \quad \leq C .
\end{aligned}
$$

By (8), then $P_{\alpha+\beta-1 ; \omega}(\mu) \in \mathcal{L} \mathcal{B}_{\omega}^{2}\left(\mathbb{B}_{n}\right)$ and $P_{\alpha+\beta-1 ; \omega}(\mu) \in \mathcal{B}_{\omega}\left(\mathbb{B}_{n}\right)$.

When $\alpha, \beta>1$, taking $g_{\mathbf{w}}(\mathbf{z})=(1-\langle\mathbf{z}, \mathbf{w}\rangle)^{1-(\alpha+\beta)}$, we have $\left\|g_{\mathbf{w}}\right\|_{\mathcal{B}_{\omega}^{\alpha, \beta}\left(\mathbb{B}_{n}\right)} \leq C$.

From Lemma 2.1, we get

$$
\left|Q_{\mu}^{\alpha, \beta ; \omega}(\mathbf{w})\right|\left(1-|\mathbf{w}|^{2}\right)^{\alpha+\beta} \leq C \quad \text { for } \mathbf{w} \in \mathbb{B}_{n} .
$$

By (8) it is obvious that $P_{\alpha+\beta-1 ; \omega}(\mu) \in \mathcal{B}_{\omega}^{\alpha, \beta}\left(\mathbb{B}_{n}\right)$.

This completes the proof of Theorem 3.1.

\section{Compactness of general Toeplitz operators}

In this section, we study the compactness of Toeplitz operators on the weighted Blochtype spaces $\mathcal{B}_{\omega}^{\alpha, \beta}\left(\mathbb{B}_{n}\right)$ in the unit ball of $\mathbb{C}^{n}$. We need the following lemma.

Lemma 4.1 Let $0<\alpha<\infty, 0 \leq \beta<\infty$ and $T_{\mu}^{\alpha, \beta ; \omega}$ be a bounded linear operator from $\mathcal{B}_{\omega}^{\alpha, \beta}\left(\mathbb{B}_{n}\right)$ into $\mathcal{B}_{\omega}^{\alpha, \beta}\left(\mathbb{B}_{n}\right)$. When $0<\alpha<1,0 \leq \beta<1$ and $\alpha+\beta<1$, then $T_{\mu}^{\alpha, \beta ; \omega}$ is compact if and only if

$$
\lim _{j \rightarrow \infty}\left\|T_{\mu}^{\alpha, \beta ; \omega} f_{j}\right\|_{\mathcal{B}_{\omega}^{\alpha, \beta}\left(\mathbb{B}_{n}\right)}=0
$$

whenever $\left(f_{j}\right)$ is a bounded sequence in $\mathcal{B}_{\omega}^{\alpha, \beta}\left(\mathbb{B}_{n}\right)$ that converges to 0 uniformly on $\overline{\mathbb{B}}_{n}$.

Proof This lemma can be proved by Montel's theorem and Lemma 2.1.

Theorem 4.1 Let $\mu$ be a positive Borel measure on $\mathbb{B}_{n}$. We have the following:

(1) if $\alpha+\beta=1$, then $T_{\mu}^{\alpha, \beta ; \omega}$ is compact on $\mathcal{B}_{\omega}^{\alpha, \beta}\left(\mathbb{B}_{n}\right)$ if and only if $P_{\alpha+\beta-1 ; \omega}(\mu) \in \mathcal{L} \mathcal{B}_{\omega ; 0}\left(\mathbb{B}_{n}\right)$ and $\mu$ is a vanishing Carleson measure;

(2) if $\alpha=\beta=1$, then $T_{\mu}^{\alpha, \beta ; \omega}$ is compact on $\mathcal{B}_{\omega}^{\alpha, \beta}\left(\mathbb{B}_{n}\right)$ if and only if $P_{\alpha+\beta-1 ; \omega}(\mu) \in \mathcal{B}_{\omega ; 0}\left(\mathbb{B}_{n}\right) \cap \mathcal{L B}_{\omega ; 0}^{2}\left(\mathbb{B}_{n}\right)$ and $\mu$ is a vanishing Carleson measure;

(3) if $\alpha>1, \beta>1$, then $T_{\mu}^{\alpha, \beta ; \omega}$ is compact on $\mathcal{B}_{\omega}^{\alpha, \beta}\left(\mathbb{B}_{n}\right)$ if and only if $P_{\alpha+\beta-1}(\mu) \in \mathcal{B}_{\omega ; 0}^{\alpha, \beta}\left(\mathbb{B}_{n}\right)$ and $\mu$ is a vanishing Carleson measure.

Proof For $\alpha+\beta \geq 1$, let $\left(g_{j}\right)$ be a sequence in $\mathcal{B}_{\omega}^{\alpha, \beta}\left(\mathbb{B}_{n}\right)$ satisfying $\left\|g_{j}\right\|_{\mathcal{B}_{\omega}^{\alpha, \beta}\left(\mathbb{B}_{n}\right)} \leq 1$ and $g_{j}$ converges to 0 uniformly as $j \rightarrow \infty$ on $\overline{\mathbb{B}}_{n}$. Suppose $f \in A^{1}\left(\mathbb{B}_{n}\right)$. By duality, we have that $T_{\mu}^{\alpha, \beta ; \omega}$ is compact on $\mathcal{B}_{\omega}^{\alpha, \beta}\left(\mathbb{B}_{n}\right)$ if and only if

$$
\lim _{j \rightarrow \infty} \sup _{\|f\|_{A^{1}\left(\mathbb{B}_{n}\right)} \leq 1}\left|\left\langle f, T_{\mu}^{\alpha, \beta ; \omega}\left(g_{j}\right)\right\rangle\right|=0 .
$$


Similarly, as in the proof of Theorem 3.1 for $s=\alpha+\beta-1$, we have

$$
\begin{aligned}
\left\langle f, T_{\mu}^{\alpha, \beta ; \omega} g_{j}\right\rangle_{s}= & c_{\alpha+\beta-1} \int_{\mathbb{B}_{n}} f(\mathbf{z}) \overline{g_{j}(\mathbf{z})}\left(1-|\mathbf{z}|^{2}\right)^{\alpha+\beta-1} d \mu(\mathbf{z}) \\
= & c_{\alpha+\beta-1} \int_{\mathbb{B}_{n}}\left[\left(I_{\mathbf{z}, \mathbf{w} ; \omega}-P_{\alpha+\beta ; \omega}\right)\left(f \bar{g}_{j}\right)\right](\mathbf{z})\left(1-|\mathbf{z}|^{2}\right)^{\alpha+\beta-1} d \mu(\mathbf{z}) \\
& +c_{\alpha+\beta-1} \int_{\mathbb{B}_{n}} P_{\alpha+\beta ; \omega}\left(f \bar{g}_{j}\right)(\mathbf{z})\left(1-|\mathbf{z}|^{2}\right)^{\alpha+\beta-1} d \mu(\mathbf{z}) \\
= & J_{1}+J_{2} .
\end{aligned}
$$

For fixed $0<\varepsilon<1$, since $\mu$ is a vanishing Carleson measure, there exists $0<\eta<1$ such that

$$
\left(1-|\mathbf{z}|^{2}\right)^{\lambda} \int_{\mathbb{B}_{n} \backslash \eta \mathbb{B}_{n}} \frac{\left(1-|\mathbf{w}|^{2}\right)^{\lambda-(\alpha+\beta)}}{|1-\langle\mathbf{z}, \mathbf{w}\rangle|^{n+2 \lambda+1-(\alpha+\beta)}} d \mu(\mathbf{w})<\varepsilon,
$$

where $\eta \mathbb{B}_{n}=\left\{\mathbf{z} \in \mathbb{C}^{n},|\mathbf{z}|<\eta\right\}$ and $\lambda-(\alpha+\beta)>-1$. For a positive constant $0<\delta<1$, as in the proof of Theorem 3.1, by Proposition 2.1, we obtain

$$
\begin{aligned}
\left|J_{1}\right|= & c_{\alpha+\beta-1} c_{\alpha+\beta}\left|\int_{\mathbb{B}_{n}} \int_{\mathbb{B}_{n}} \frac{\left(\overline{g_{j}(\mathbf{z})}-\overline{g_{j}(\mathbf{w})}\right) f(\mathbf{w})\left(1-|\mathbf{w}|^{2}\right)^{\alpha+\beta}\left(1-|\mathbf{z}|^{2}\right)^{\alpha+\beta-1}}{(1-\langle\mathbf{z}, \mathbf{w}\rangle)^{n+\alpha+1}\left(1-\left|\varphi_{\mathbf{w}}(\mathbf{z})\right|^{2}\right)^{\beta} \omega(1-|\mathbf{z}|)} d \nu(\mathbf{w}) d \mu(\mathbf{z})\right| \\
= & c_{\alpha+\beta-1} c_{\alpha+\beta} \mid \int_{\mathbb{B}_{n}} f(\mathbf{w})\left(1-|\mathbf{w}|^{2}\right)^{\alpha+\beta} \\
& \times \int_{\mathbb{B}_{n}} \frac{\left(\overline{g_{j}(\mathbf{z})}-\overline{g_{j}(\mathbf{w})}\right)\left(1-|\mathbf{z}|^{2}\right)^{\alpha+\beta-1}}{(1-\langle\mathbf{z}, \mathbf{w}\rangle)^{n+\alpha+1}\left(1-\mid \varphi_{\mathbf{w}}(\mathbf{z})^{2}\right)^{\beta} \omega(1-|\mathbf{z}|)} d \mu(\mathbf{z}) d v(\mathbf{w}) \mid \\
\leq & c_{\alpha+\beta-1} c_{\alpha+\beta} \int_{\mathbb{B}_{n} \backslash \delta \mathbb{B}_{n}}|f(\mathbf{w})|\left(1-|\mathbf{w}|^{2}\right)^{\alpha+\beta} \\
& \times \int_{\mathbb{B}_{n}} \frac{\left|g_{j}(\mathbf{z})-g_{j}(\mathbf{w})\right|\left(1-|\mathbf{z}|^{2}\right)^{\alpha+\beta-1}}{|1-\langle\mathbf{z}, \mathbf{w}\rangle|^{n+\alpha+1}\left(1-\mid \varphi_{\mathbf{w}}(\mathbf{z})^{2}\right)^{\beta} \omega(1-|\mathbf{z}|)} d \mu(\mathbf{z}) d \nu(\mathbf{w}) \\
& +c_{\alpha+\beta-1} c_{\alpha+\beta} \int_{\delta \mathbb{B}_{n}}|f(\mathbf{w})|\left(1-|\mathbf{w}|^{2}\right)^{\alpha+\beta} \\
& \times \int_{\mathbb{B}_{n}} \frac{\left|g_{j}(\mathbf{z})-g_{j}(\mathbf{w})\right|\left(1-|\mathbf{z}|^{2}\right)^{\alpha+\beta-1}}{|1-\langle\mathbf{z}, \mathbf{w}\rangle|^{n+\alpha+1}\left(1-\left|\varphi_{\mathbf{w}}(\mathbf{z})\right|^{2}\right)^{\beta} \omega(1-|\mathbf{z}|)} d \mu(\mathbf{z}) d \nu(\mathbf{w}) \\
= & L_{1}+L_{2} .
\end{aligned}
$$

Since $g_{j} \rightarrow 0$ as $j \rightarrow \infty$ on compact subsets of $\mathbb{B}_{n}$, we can choose $j$ big enough so that

$$
|f(\mathbf{w})|\left(1-|\mathbf{w}|^{2}\right)^{\alpha+\beta}<\varepsilon
$$

Therefore,

$$
\begin{aligned}
L_{2} & \leq \varepsilon C \int_{\delta \mathbb{B}_{n}} \int_{\mathbb{B}_{n}} \frac{\left|g_{j}(\mathbf{z})-g_{j}(\mathbf{w})\right|\left(1-|\mathbf{z}|^{2}\right)^{\alpha+\beta-1}}{|1-\langle\mathbf{z}, \mathbf{w}\rangle|^{n+\alpha+1}\left(1-\left|\varphi_{\mathbf{w}}(\mathbf{z})\right|^{2}\right)^{\beta} \omega(1-|\mathbf{z}|)} d \mu(\mathbf{z}) d \nu(\mathbf{w}) \\
& \leq \varepsilon C\left\|g_{j}\right\|_{\mathcal{B}_{\omega}^{\alpha, \beta}\left(\mathbb{B}_{n}\right)^{.}}
\end{aligned}
$$


Now, taking $\delta$ such that $1-\left[\varepsilon(1-\eta)^{n+1+\lambda}\right]^{\frac{1}{\lambda}} \leq \delta<1$, then

$$
\begin{aligned}
L_{1} \leq & C\left\|g_{j}\right\|_{\mathcal{B}_{\omega}^{\alpha, \beta}\left(\mathbb{B}_{n}\right)} \int_{\mathbb{B}_{n} \backslash \delta \mathbb{B}_{n}}|f(\mathbf{w})|\left(1-|\mathbf{w}|^{2}\right)^{\lambda} \int_{\mathbb{B}_{n}} \frac{\left(1-|\mathbf{z}|^{2}\right)^{\lambda-(\alpha+\beta)}}{|1-\langle\mathbf{z}, \mathbf{w}\rangle|^{n+2 \lambda+1-(\alpha+\beta)}} d \mu(\mathbf{z}) d \nu(\mathbf{w}) \\
\leq & C \int_{\mathbb{B}_{n} \backslash \delta \mathbb{B}_{n}}|f(\mathbf{w})|\left(1-|\mathbf{w}|^{2}\right)^{\lambda} \int_{\mathbb{B}_{n} \backslash \eta \mathbb{B}_{n}} \frac{\left(1-|\mathbf{z}|^{2}\right)^{\lambda-(\alpha+\beta)}}{|1-\langle\mathbf{z}, \mathbf{w}\rangle|^{n+2 \lambda+1-(\alpha+\beta)}} d \mu(\mathbf{z}) d \nu(\mathbf{w}) \\
& +C \int_{\mathbb{B}_{n} \backslash \delta \mathbb{B}_{n}}|f(\mathbf{w})|\left(1-|\mathbf{w}|^{2}\right)^{\lambda} \int_{\eta \mathbb{B}_{n}} \frac{\left(1-|\mathbf{z}|^{2}\right)^{\lambda-(\alpha+\beta)}}{|1-\langle\mathbf{z}, \mathbf{w}\rangle|^{n+2 \lambda+1-(\alpha+\beta)}} d \mu(\mathbf{z}) d \nu(\mathbf{w}) \\
\leq & C \varepsilon \int_{\mathbb{B}_{n} \backslash \delta \mathbb{B}_{n}}|f(\mathbf{w})| d \nu(\mathbf{w})+C \int_{\mathbb{B}_{n} \backslash \delta \mathbb{B}_{n}}|f(\mathbf{w})| \frac{(1-\delta)^{\lambda}}{(1-\eta)^{n+1+\lambda}} d \nu(\mathbf{w}) \\
\leq & C \varepsilon\|f\|_{A^{1}\left(\mathbb{B}_{n}\right)} .
\end{aligned}
$$

Hence $\left|J_{1}\right|<C \varepsilon$, where $C$ does not depend on $f(\mathbf{z})$, and so

$$
\lim _{j \rightarrow \infty} \sup _{\|f\|_{A^{1}\left(\mathbb{B}_{n}\right)} \leq 1}\left|J_{1}\right|=0
$$

Thus, $T_{\mu}^{\alpha, \beta ; \omega}$ is compact on $\mathcal{B}_{\omega}^{\alpha, \beta}\left(\mathbb{B}_{n}\right)$ if and only if

$$
\lim _{j \rightarrow \infty} \sup _{\|f\|_{A^{1}\left(\mathbb{B}_{n}\right)} \leq 1}\left|J_{2}\right|=0
$$

Again, as in the proof of Theorem 3.1, we have

$$
\left|J_{2}\right| \leq C \int_{\mathbb{B}_{n}}\|f\|_{A^{1}\left(\mathbb{B}_{n}\right)}\left(1-|\mathbf{w}|^{2}\right)^{\alpha+\beta}|g(\mathbf{w})| Q_{\mu}^{\alpha, \beta ; \omega}(\mathbf{w}) d \nu(\mathbf{w}) .
$$

From (8) it is easy to see that

(1) if $\alpha+\beta=1$ and $P_{\alpha+\alpha-1 ; \omega}(\mu) \in \mathcal{L} \mathcal{B}_{\omega, 0}^{\alpha, \beta}\left(\mathbb{B}_{n}\right)$, then

$$
\lim _{|\mathbf{w}| \rightarrow 1}\left(1-|\mathbf{w}|^{2}\right) Q_{\mu}^{\alpha, \beta ; \omega}(\mathbf{w})\left(\ln \frac{2}{1-|\mathbf{w}|^{2}}\right)=0 ;
$$

(2) if $\alpha=\beta=1, P_{\alpha+\alpha-1 ; \omega}(\mu) \in \mathcal{B}_{\omega ; 0}\left(\mathbb{B}_{n}\right) \cap \mathcal{L B}_{\omega ; 0}^{2}\left(\mathbb{B}_{n}\right)$, then

$$
\lim _{|\mathbf{w}| \rightarrow 1}\left(1-|\mathbf{w}|^{2}\right) Q_{\mu}^{\alpha, \beta ; \omega}(\mathbf{w})=0
$$

and

$$
\lim _{|\mathbf{w}| \rightarrow 1}\left(1-|\mathbf{w}|^{2}\right)^{2} Q_{\mu}^{\alpha, \beta ; \omega}(\mathbf{w})\left(\ln \frac{2}{1-|\mathbf{w}|^{2}}\right)=0 ;
$$

(3) if $\alpha>1, \beta>1$, and $P_{\alpha+\alpha-1 ; \omega}(\mu) \in \mathcal{B}_{\omega ; 0}^{\alpha, \beta}\left(\mathbb{B}_{n}\right)$, then

$$
\lim _{|\mathbf{w}| \rightarrow 1}\left(1-|\mathbf{w}|^{2}\right)^{\alpha+\beta} Q_{\mu}^{\alpha, \beta ; \omega}(\mathbf{w})=0 .
$$


Combined with $g_{j} \rightarrow 0$ as $j \rightarrow \infty$ on compact subsets of $\mathbb{B}_{n}$, we have

$$
\lim _{j \rightarrow \infty} \sup _{\|f\|_{A^{1}\left(\mathbb{B}_{n}\right)} \leq 1}\left|J_{2}\right|=0
$$

Therefore,

$$
\lim _{j \rightarrow \infty}\left\|T_{\mu}^{\alpha, \beta ; \omega} g_{j}\right\|_{\mathcal{B}_{\omega}^{\alpha, \beta}\left(\mathbb{B}_{n}\right)}=0
$$

which implies that $T_{\mu}^{\alpha, \beta ; \omega}$ is a compact operator.

Next assume that $T_{\mu}^{\alpha, \beta ; \omega}$ is a compact operator on $\mathcal{B}_{\omega}^{\alpha, \beta}\left(\mathbb{B}_{n}\right)$. Again, as in the proof of Theorem 3.1, we take

$$
f_{\mathbf{w}}(\mathbf{z})=\frac{\left(1-|\mathbf{w}|^{2}\right)^{t}}{(1-\langle\mathbf{z}, \mathbf{w}\rangle)^{n+t+1}} \quad \text { for } t>0 .
$$

We know that $\left\|f_{\mathbf{w}}\right\|_{A^{1}\left(\mathbb{B}_{n}\right)} \leq C$. On the other hand, take

$$
g_{\mathbf{w}}(\mathbf{z})=\frac{\left(1-|\mathbf{w}|^{2}\right)^{n+2+t-(\alpha+\beta)}}{(1-\langle\mathbf{z}, \mathbf{w}\rangle)^{n+t+1}} ; \quad \varphi_{\mathbf{w}}(\mathbf{z}) \equiv 1 \quad \text { and } \quad \omega(1-|\mathbf{z}|) \equiv 1 \quad \text { for } t>0 .
$$

Then $\left\|g_{\mathbf{w}}\right\|_{\mathcal{B}_{\omega}^{\alpha, \beta}\left(\mathbb{B}_{n}\right)} \leq C$ and $g_{\mathbf{w}} \rightarrow 0$ uniformly on compact subsets of $\mathbb{B}_{n}$, as $|\mathbf{w}| \rightarrow 1$,

$$
\begin{aligned}
& \left|\left\langle f, T_{\mu}^{\alpha, \beta ; \omega} g\right\rangle_{s}\right| \\
& \quad=c_{\alpha+\beta-1}\left(1-|\mathbf{w}|^{2}\right)^{n+2+2 t-(\alpha+\beta)} \int_{\mathbb{B}_{n}} \frac{\left(1-|\mathbf{z}|^{2}\right)^{\alpha+\beta-1} d \mu(\mathbf{z})}{|1-\langle\mathbf{z}, \mathbf{w}\rangle|^{2(n+t+1)}} \\
& \leq C\left\|f_{\mathbf{w}}\right\|_{A^{1}\left(\mathbb{B}_{n}\right)}\left\|T_{\mu}^{\alpha, \beta} g_{\mathbf{w}}\right\|_{\mathcal{B}^{\alpha, \beta}\left(\mathbb{B}_{n}\right)^{.}}
\end{aligned}
$$

From Lemma 4.1, we have

$$
\lim _{|\mathbf{w}| \rightarrow 1}\left\|f_{\mathbf{w}}\right\|_{A^{1}\left(\mathbb{B}_{n}\right)}\left\|T_{\mu}^{\alpha, \beta ; \omega} g_{\mathbf{w}}\right\|_{\mathcal{B}_{\omega}^{\alpha, \beta}\left(\mathbb{B}_{n}\right)}=0, \quad \forall \mathbf{w} \in \mathbb{B}_{n}
$$

This implies that $\mu$ is a vanishing Carleson measure on $\mathbb{B}_{n}$.

Next let

$$
f_{\mathbf{w}}(\mathbf{z})=\frac{\left(1-|\mathbf{w}|^{2}\right)^{\alpha+\beta}}{(1-\langle\mathbf{z}, \mathbf{w}\rangle)^{n+\alpha+\beta+1}} .
$$

Then, we have $\left\|f_{\mathbf{w}}\right\|_{A^{1}\left(\mathbb{B}_{n}\right)} \leq C$. Let $\left\{g_{j}\right\}$ be a bounded sequence in $\mathcal{B}_{\omega}^{\alpha, \beta}\left(\mathbb{B}_{n}\right)$ that converges to zero uniformly as $j \rightarrow \infty$ on $\overline{\mathbb{B}}_{n}$. By the compactness of $T_{\mu}^{\alpha, \beta ; \omega}$, we have

$$
\begin{aligned}
0 & =\lim _{j \rightarrow \infty} J_{2}=\lim _{j \rightarrow \infty} c_{\alpha+\beta} \int_{\mathbb{B}_{n}} f_{\mathbf{w}}(\mathbf{z})\left(1-|\mathbf{z}|^{2}\right)^{\alpha+\beta} \overline{g_{j}(\mathbf{z}) Q_{\mu}^{\alpha, \beta ; \omega}(\mathbf{z})} d v(\mathbf{z}) \\
& =\lim _{j \rightarrow \infty} c_{\alpha+\beta}\left(1-|\mathbf{w}|^{2}\right)^{\alpha+\beta} \int_{\mathbb{B}_{n}} \frac{\left(1-|\mathbf{z}|^{2}\right)^{\alpha+\beta} \overline{g_{j}(\mathbf{z}) Q_{\mu}^{\alpha, \beta ; \omega}(\mathbf{z})}}{(1-\langle\mathbf{z}, \mathbf{w}\rangle)^{n+\alpha+\beta+1}} d v(\mathbf{z}) \\
& =\lim _{j \rightarrow \infty}\left(1-|\mathbf{w}|^{2}\right)^{\alpha+\beta} \overline{g_{j}(\mathbf{w}) Q_{\mu}^{\alpha, \beta ; \omega}(\mathbf{w})} .
\end{aligned}
$$


When $\alpha+\beta=1$, taking

$$
g_{\mathbf{w}}(\mathbf{z})=\left(\ln \frac{2}{1-\langle\mathbf{z}, \mathbf{w}\rangle}\right)^{2}\left(\ln \frac{1}{1-|\mathbf{w}|^{2}}\right)^{-1} ; \quad \varphi_{\mathbf{w}}(\mathbf{z}) \equiv 1 \quad \text { and } \quad \omega(1-|\mathbf{z}|) \equiv 1,
$$

with $|\mathbf{w}| \geq \frac{1}{2}$, we have $P_{\alpha+\beta-1 ; \omega}(\mu) \in \mathcal{L B}_{\omega ; 0}\left(\mathbb{B}_{n}\right)$.

When $\alpha=\beta=1$, taking

$$
g_{\mathbf{w}}(\mathbf{z})=\frac{1-|\mathbf{w}|^{2}}{(1-\langle\mathbf{z}, \mathbf{w}\rangle)^{2}}+\ln \frac{2}{1-\langle\mathbf{z}, \mathbf{w}\rangle} ; \quad \varphi_{\mathbf{w}}(\mathbf{z}) \equiv 1 \quad \text { and } \quad \omega(1-|\mathbf{z}|) \equiv 1,
$$

we have $P_{\alpha+\beta-1 ; \omega}(\mu) \in \mathcal{L B}_{\omega}^{2}\left(\mathbb{B}_{n}\right) \cap \mathcal{B}_{\omega}\left(\mathbb{B}_{n}\right)$.

Finally, when $\alpha, \beta>1$, take

$$
g_{\mathbf{w}}(\mathbf{z})=\frac{1-|\mathbf{w}|^{2}}{(1-\langle\mathbf{z}, \mathbf{w}\rangle)^{\alpha+\beta}} ; \quad \varphi_{\mathbf{w}}(\mathbf{z}) \equiv 1 \quad \text { and } \quad \omega(1-|\mathbf{z}|) \equiv 1
$$

Then, it is obvious that $P_{\alpha+\beta-1 ; \omega}(\mu) \in \mathcal{B}_{\omega}^{\alpha, \beta}\left(\mathbb{B}_{n}\right)$.

This completes the proof of Theorem 4.1.

Remark 4.1 It is still an open problem to study the properties of radial Toeplitz operators on the studied spaces of this paper. For more information on radial Toeplitz operators, we refer to $[23,24]$.

\section{Competing interests}

The author declares that they have no competing interests.

Received: 17 October 2012 Accepted: 17 April 2013 Published: 9 May 2013

\section{References}

1. Hahn, KT, Choi, KS: Weighted Bloch spaces in $\mathbb{C}^{n}$. J. Korean Math. Soc. 35, 171-189 (1998)

2. Li, S, Wulan, H: Characterizations of $\alpha$-Bloch spaces on the ball. J. Math. Anal. Appl. 343(1), 58-63 (2008)

3. Nowak, M: Bloch and Möbius invariant Besov spaces on the unit ball of $\mathbb{C}^{n}$. Complex Var. Theory Appl. 44, 1-12 (2001)

4. Zhu, K: Bloch-type spaces of analytic functions. Rocky Mt. J. Math. 23, 1143-1177 (1993)

5. Zhu, K: Spaces of Holomorphic Functions in the Unit Ball. Springer, New York (2004)

6. Zhu, K: Positive Toeplitz operators on weighted Bergman spaces of bounded symmetric domains. J. Oper. Theory 20, 329-357 (1988)

7. Zhu, K: Operator Theory in Function Spaces. Dekker, New York (1990)

8. Wu, Z, Zhao, R, Zorboska, N: Toeplitz operators on Bloch-type spaces. Proc. Am. Math. Soc. 134, 3531-3542 (2006)

9. Wang, X, Liu, T: Toeplitz operators on Bloch-type spaces in the unit ball of $\mathbb{C}^{n}$. J. Math. Anal. Appl. 368, $727-735$ (2010)

10. Wu, Z, Zhao, R, Zorboska, N: Toeplitz operators on analytic Besov spaces. Integral Equ. Oper. Theory 60, 435-449 (2008)

11. El-Sayed Ahmed, A, Bakhit, MA: Properties of Toeplitz operators on some holomorphic Banach function spaces. J. Funct. Spaces Appl. 2012, Article ID 517689 (2012)

12. Das, N, Sahoo, M: Positive Toeplitz operators on the Bergman space. Ann. Funct. Anal. 4(2), 171-182 (2013)

13. Englis, M: Toeplitz operators and localization operators. Trans. Am. Math. Soc. 361, 1039-1052 (2009)

14. Englis, M: Toeplitz operators and weighted Bergman kernels. J. Funct. Anal. 255, 1419-1457 (2008)

15. Perälä, A: Toeplitz operators on Bloch-type spaces and classes of weighted Sobolev distributions. Integral Equ. Oper. Theory 71(1), 113-128 (2011)

16. Sánchez-Nungaray, A, Vasilevski, N: Toeplitz operators on the Bergman spaces with pseudodifferential defining symbols. In: Karlovich, Yl, Rodino, L, Silbermann, B, Spitkovsky, IM (eds.) Operator Theory, Pseudo-Differential Equations, and Mathematical Physics. Operator Theory: Advances and Applications, vol. 228, pp. 355-374. Birkhäuser, Basel (2013)

17. Vasilevski, NL: Commutative Algebras of Toeplitz Operators on the Bergman Space. Operator Theory: Advances and Applications, vol. 185, xxix. Birkhäuser, Basel (2008)

18. El-Sayed Ahmed, A: Criteria for functions to be weighted Bloch. J. Comput. Anal. Appl. 11(2), 252-262 (2009)

19. Zhu, K: Multipliers of BMO in the Bergman metric with applications to Toeplitz operators. J. Funct. Anal. 87, 31-50 (1989)

20. Ren, G, Tu, C: Bloch spaces in the unit ball of $\mathbb{C}^{n}$. Proc. Am. Math. Soc. 133, 719-726 (2005) 
21. Zhao, R: A characterization of Bloch-type spaces on the unit ball of $\mathbb{C}^{n}$. J. Math. Anal. Appl. 330, 291-297 (2007)

22. Rudin, W: Function Theory in the Unit Ball of $\mathbb{C}^{n}$. Springer, New York (1980)

23. Grudsky, S, Maximenko, E, Vasilevski, NL: Radial Toeplitz operators on the unit ball and slowly oscillating sequences. Commun. Math. Anal. 14(2), 77-94 (2013)

24. Zhou, ZH, Chen, WL, Dong, XT: The Berezin transform and radial operators on the Bergman space of the unit ball. Complex Anal. Oper. Theory 7(1), 313-329 (2013)

doi:10.1186/1029-242X-2013-237

Cite this article as: El-Sayed Ahmed: General Toeplitz operators on weighted Bloch-type spaces in the unit ball of $\mathbb{C}^{n}$ Journal of Inequalities and Applications 2013 2013:237.

Submit your manuscript to a SpringerOpen ${ }^{\circ}$ journal and benefit from:

- Convenient online submission

- Rigorous peer review

Immediate publication on acceptance

- Open access: articles freely available online

- High visibility within the field

- Retaining the copyright to your article

Submit your next manuscript at $>$ springeropen.com 